\title{
Dirac Operator on Complex Manifolds and Supersymmetric Quantum Mechanics
}

\author{
E. A. Ivanov ${ }^{*, 1}$, A. V. Smilga ${ }^{\dagger, 2}$ \\ * Bogoliubov Laboratory of Theoretical Physics \\ JINR, 141980 Dubna, Russia \\ † SUBATECH, Université de Nantes, \\ 4 rue Alfred Kastler, BP 20722, Nantes 44307, France; \\ On leave of absence from ITEP, Moscow, Russia
}

\begin{abstract}
We explore a simple $\mathcal{N}=2$ SQM model describing the motion over complex manifolds in external gauge fields. The nilpotent supercharge $Q$ of the model can be interpreted as a (twisted) exterior holomorphic derivative, such that the model realizes the twisted Dolbeault complex. The sum $Q+\bar{Q}$ can be interpreted as the Dirac operator: the standard Dirac operator if the manifold is Kähler and the Dirac operator involving certain particular extra torsions for a generic complex manifold. Focusing on the Kähler case, we give new simple physical proofs of the two mathematical facts: (i) the equivalence of the twisted Dirac and twisted Dolbeault complexes and (ii) the Atiyah-Singer theorem.
\end{abstract}

\footnotetext{
1 eivanov@theor.jinr.ru

2 smilga@subatech.in2p3.fr
} 


\section{Introduction}

Complexes are the algebraic objects associated with smooth manifolds and studied in differential geometry [1]. The most known is the de Rham complex involving the exterior derivative $d$ and the Hermitian-conjugate operator $d^{\dagger}=(-1)^{D p+D+1} \star d \star$ acting on the space of $p$-forms ( $D$ is the dimension of the manifold and $\star$ is the Hodge duality operator). The operators $d$ and $d^{\dagger}$ are nilpotent, while their anticommutator $\left\{d, d^{\dagger}\right\}$ coincides with the covariant Laplacian acting on the forms. The other important complexes are the Dolbeault complex, which is defined on complex manifolds and involves holomorphic exterior derivative $\partial$ and its hermitian conjugate $\partial^{\dagger}$, and the Dirac complex associated with the Dirac operator. The complexes may be twisted by adding background Abelian or non-Abelian gauge fields 1 .

An important characteristics of all these complexes are their indices. The index of an elliptic operator $2 \mathcal{O}$ can be defined if the whole Hilbert space of objects (states) where it acts can be divided into two subspaces (call them $\mathcal{H}_{L}$ and $\mathcal{H}_{R}$ ) and there are symmetry operators (commuting with $\mathcal{O}$ ) that transform a state from $\mathcal{H}_{L}$ into a state from $\mathcal{H}_{R}$ and a state from $\mathcal{H}_{R}$ into a state from $\mathcal{H}_{L}$.

In such case, one can always define nilpotent projections: an operator that brings a state from $\mathcal{H}_{L}$ into a state from $\mathcal{H}_{R}$ and annihilates any state from $\mathcal{H}_{R}$, and its Hermitian conjugate: the operator bringing a state from $\mathcal{H}_{R}$ into $\mathcal{H}_{L}$ and annihilating the states from $\mathcal{H}_{L}$. The anticommutator of these nilpotent projections is a symmetry operator too. In the simplest case, it coincides with $\mathcal{O}$. Then all eigenstates of $\mathcal{O}$ with nonzero eigenvalues are double degenerate (take an eigenstate from $\mathcal{H}_{L}$ and act upon it by a symmetry operator). It is not true for zero eigenvalues. The index is then defined as the difference between the number of states in the kernel of $\mathcal{O}$ belonging to $\mathcal{H}_{L}$ and such a number for $\mathcal{H}_{R}$.

For example, for the de Rham complex, $\mathcal{O}=-\triangle_{\text {cov }}$, and the Hilbert space of all relevant forms can be divided into the subsets of even and odd forms. The relevant symmetry operators are $d$ and $d^{\dagger}$. For the Dirac complex $3^{3}, \mathcal{O}=-\mathbb{D}^{2}$, the Hilbert space of all spinors can be subdivided into the left-handed spinors and the righthanded ones. The symmetry operators are $\not{D}$ and $\not{D} \gamma^{D+1}$ (the index of $\mathcal{O}$ coinciding with the index of $\not \mathcal{D}$ can be defined only for even-dimensional manifolds where $\gamma^{D+1}$, a multidimensional generalization of $\gamma^{5}$, can be defined).

The indices have beautiful integral representations. Consider, e.g., the 2-dim Dirac operator in an external Abelian field on the plane. Its index coincides with the magnetic flux,

$$
I_{\not{D}}=\frac{1}{2 \pi} \int B d^{2} x
$$

\footnotetext{
1 There are two parallel terminological systems: physical and mathematical. For example, what a physicist calls Abelian gauge field is called connection on a line bundle by a mathematician. We will mostly use the physical terminology.

${ }^{2}$ Recall that elliptic operators are invertible differential operators that generalize the Laplace operator (and of course include it as a particular case).

${ }^{3}$ The sign is chosen so that the operator $\mathcal{O}$ is positive-definite with $\not \mathcal{D}=\mathcal{D}_{M} \gamma^{M}$ and Hermitian $\gamma^{M}$.
} 
The integral representations for all indices were systematically derived by Atiyah and Singer [2, 3, 4, 5]. In their derivation, they used the so called heat kernel method based on the semiclassical ( small $\beta$ ) expansion of the matrix element $\left\langle x\left|\Gamma e^{-\beta \mathcal{O}}\right| x\right\rangle$, where $\Gamma$ is the grading operator distinguishing between $\mathcal{H}_{L}$ and $\mathcal{H}_{R}$, such that $\Gamma \Psi=\Psi$ when $\Psi \in \mathcal{H}_{L}$ and $\Gamma \Psi=-\Psi$ when $\Psi \in \mathcal{H}_{R}$. (For good pedagogical explanations, see e.g. [6, 7].)

An interesting, from the physical viewpoint, modification of this method is based on the observation that the indices of elliptic (Euclidean) operators are associated via the level crossing picture with the anomalies of certain Minkowski space currents [8, 9, 10]. For example, the index (1.1) is associated with the anomalous divergence of the 2-dim axial current $J_{M}=\bar{\psi} \gamma_{M} \sigma^{3} \psi$,

$$
\partial_{M} J_{M}=\frac{1}{4 \pi} \epsilon_{M N} F_{M N}
$$

One of the ways to derive (1.2) is to regularize the current by the Schwinger splitting $J_{M} \rightarrow J_{M}(\epsilon)=\bar{\psi}(x+\epsilon) \gamma_{M} \sigma^{3} \psi(x-\epsilon)$ and calculate then the Euclidean fermion propagator in external Abelian field $\langle\psi(x-\epsilon) \bar{\psi}(x+\epsilon)\rangle_{A}$ using the Fock-Schwinger gauge technique [11].

For the index (1.1), the heat kernel calculation is rather explicit, but it is much more intricate in more complicated cases of mathematical and physical interest.

Back in 1981, Witten noticed [12, 13 that this set of mathematical problems has a beautiful physical interpretation: the operators $\mathcal{O}$ can be viewed of as Hamiltonians of certain supersymmetric quantum mechanics (SQM) systems, while the nilpotent projections discussed above are interpreted as supercharges. The index of $\mathcal{O}$ coincides then with the Witten index of the corresponding SQM system,

$$
I=\operatorname{Tr}\left\{\Gamma e^{-\beta H}\right\}=\operatorname{Tr}\left\{(-1)^{F} e^{-\beta H}\right\}
$$

where $\beta$ is a parameter having the meaning of inverse temperature and $F$ is an operator that commutes with $H$ and has even eigenvalues for the states from $\mathcal{H}_{L}$ and odd eigenvalues for the states from $\mathcal{H}_{R}$. Physically, $F$ is interpreted as the fermion number. Due to degeneracy between the excited states in $\mathcal{H}_{L}$ and $\mathcal{H}_{R}$, the index does not depend on $\beta$.

Now, the r.h.s. of (1.3) has a functional integral representation. For small $\beta$, this functional integral can be evaluated by semiclassical methods. As a result, the Atiyah-Singer integral theorems are reproduced. This program was carried out in [14, 15, 16] (see also, e.g. [17, 18, 19]).

In our paper, we concentrate on complex manifolds and study the $\mathcal{N}=2 \mathrm{SQM}$ mode 4 which, in our opinion, is most appropriate for calculating the relevant indices. Its classical $\mathcal{N}=2$ superfield Lagrangian is a particular case of the general Lagrangian given in [20]. In the Kähler case, this SQM model is reduced to the model considered in [14, 15, 16], whereas in a generic complex case its Lagrangian is different. Also,

\footnotetext{
${ }^{4}$ Following the convention adopted now by the most practitioners of SQM, $\mathcal{N}$ counts the number of real supercharges. Thus, the minimal interesting case where supersymmetry (double degeneracy of all excited levels) is present in the spectrum of the Hamiltonian corresponds to $\mathcal{N}=2$.
} 
in the Kähler case, our approach differs from the approach in Ref. [14, 15, 16] by the choice of supercharges. Instead of the supercharges $\not{D}$ and $\not{D} \gamma^{D+1}$ that realize the supersymmetry algebra for any even-dimensional manifold, we use the (Hermitian) supercharges

$$
\not D D=i \gamma^{M} \mathcal{D}_{\mathcal{M}}, \quad S=I_{N}^{M} \gamma^{N} \mathcal{D}_{M},
$$

where $I_{N}^{M}$ is the complex structure tensor satisfying the properties

$$
I_{M N}=-I_{N M}, \quad I_{N}^{M} I_{P}^{N}=-\delta_{P}^{M} .
$$

The existence of the supercharge $S$ (such that $S^{2}=H$ and $\{S, \not{D}\}=0$ ) is specific for Kähler manifolds [21]. The supercharges (1.4) are naturally obtained in our superfield framework as a real and imaginary part of a certain complex nilpotent supercharge.

After fixing the complex geometry notations in Sect. 2, we present our model in Sect. 3. In Sect. 4, we show how this $\mathcal{N}=2 \mathrm{SQM}$ model can, in the Kähler case, be completed to the extended $\mathcal{N}=4 \mathrm{SQM}$ model. In Sect. 5, we give a geometric interpretation (1.4) for the Nöther supercharges derived in Sect. 3. We also observe that the nilpotent supercharge $\not{D}+S$ can be interpreted as the (twisted) operator of the holomorphic exterior derivative. This allows one to prove, in a rather manifest way, the known mathematical fact: for Kähler manifolds, the twisted Dirac complex and the twisted Dolbeault complex are equivalent. Sect. 6 is devoted to the functional integral derivation of the Atiyah-Singer theorem for the Dirac operator. The derivation is similar in spirit to the derivation in Ref. [14, 15, 16], but we do it in a much more detailed way focusing on the Kähler case.

Note that the first e-print version of our paper appeared in archive more than 1.5 years ago [22]. Since then, it inspired a few papers where its results were further applied and extended [23, 24, 25, 26]. We will comment on these subsequent developments in the proper places below. We will also make more precise some statements of [22] and add, in the last Section, a short account of the new results.

\section{Complex geometry}

Let us start with recalling some mathematical facts on complex geometry adapted for immediate use in the next section where the relevant SQM system will be introduced.

We assume the manifold to be even-dimensional of dimension $D=2 n$ and described by complex coordinates $z^{N}=\left(z^{j}, \bar{z}^{\bar{k}}\right)$. The metric is assumed to have the Hermitian form $d s^{2}=2 h_{j \bar{k}} d z^{j} d \bar{z}^{\bar{k}}$. In other word $₫$,

$$
g_{M N}=\left(\begin{array}{cc}
0 & h_{j \bar{k}} \\
h_{k \bar{j}} & 0
\end{array}\right) .
$$

\footnotetext{
${ }^{5}$ Such a manifold is not necessarily complex in the precise mathematical sense. The genuine complex manifold is required to be divisible in several charts such that the metric has the form (2.1) in each chart and the coordinates $\left(z^{j}, \bar{z}^{\bar{k}}\right)$ in such different overlapping charts are expressed through each other by means of holomorphic functions. Thus, $S^{4}$ (in contrast to $S^{2}$ ) is not a complex manifold even though its metric can be represented as in Eq.2.1) in both the northern and the southern hemispheres. Sill notice that the requirement for the metric to be representable locally in the form (2.1) is nontrivial and singles out some subset of even-dimensional manifolds.
} 
The covariant derivatives are defined as

$$
\nabla_{M} V^{N}=\partial_{M} V^{N}+\hat{\Gamma}_{M P}^{N} V^{P}, \quad \nabla_{M} V_{N}=\partial_{M} V_{N}-\hat{\Gamma}_{M N}^{P} V_{P}
$$

(note the order of indices in $\hat{\Gamma}_{M P}^{N}, \hat{\Gamma}_{M N}^{P}$ ). They involves generically the affine connections

$$
\hat{\Gamma}_{N K}^{M}=\Gamma_{N K}^{M}+\frac{1}{2} g^{M L} C_{L N K},
$$

where $\Gamma_{N K}^{M}$ are the standard Christoffel symbols for the metric $g_{M N}$ and $C_{L N K}$ is the totally antisymmetric torsion tensor. We will also use the notation

$$
\nabla \psi^{M}=\dot{\psi}^{M}+\dot{z}^{N} \hat{\Gamma}_{N L}^{M} \psi^{L}
$$

In the following, we will stick to a special form of the torsion tensor with the nonvanishing components

$$
C_{j k \bar{l}}=\partial_{k} h_{j \bar{l}}-\partial_{j} h_{k \bar{l}}, \quad C_{\bar{j} \bar{k} l}=\left(C_{j k \bar{l}}\right)^{*}=\partial_{\bar{k}} h_{l \bar{j}}-\partial_{\bar{j}} h_{l \bar{k}}
$$

(and those obtained from them by the cyclic permutation of indices). In real notations, this can be represented as [20]

$$
C_{J K L}=I_{J}^{P} I_{K}^{R} I_{L}^{T}\left(\nabla_{P} I_{R T}+\nabla_{R} I_{T P}+\nabla_{T} I_{P R}\right)
$$

The connection (2.3) with the torsion (2.5, 2.6) is known to mathematicians as Bismut connection [27] (see also [28] ) defined as a connection with respect to which both the metric tensor $g_{M N}$ and the complex structure tensor $I_{M N}$ are covariantly constant while the torsion tensor $C_{J K L}$ is completely antisymmetric

The torsion (2.6) has, generically, a non-zero curl, $\partial_{[M} C_{J K L]} \neq 06$.

Non-vanishing components of $\Gamma_{M, N P}$ and $\hat{\Gamma}_{M, N P}$ are

$$
\begin{aligned}
& \Gamma_{\bar{m}, n p}=\frac{1}{2}\left(\partial_{n} h_{p \bar{m}}+\partial_{p} h_{n \bar{m}}\right), \quad \Gamma_{m, n \bar{p}}=\Gamma_{m, \bar{p} n}=\frac{1}{2} C_{m n \bar{p}}, \quad \text { (and c.c.) }, \\
& \hat{\Gamma}_{\bar{m}, n p}=\partial_{p} h_{n \bar{m}}, \quad \hat{\Gamma}_{m, n \bar{p}}=C_{m n \bar{p}}, \quad \hat{\Gamma}_{m, \bar{p} n}=0, \quad \text { (and c.c.) } .
\end{aligned}
$$

The last identity means that, after a parallel transport with the Bismut affine connections, an (anti)holomorphic vector is transformed into an (anti)holomorphic vector: the holonomy group is $U(n)$ rather than $S O(2 n)$. As is seen from the first line in (2.7), this property does not hold for usual torsionless covariant derivatives for a generic complex manifold.

Let us introduce complex vielbeins as

$$
\begin{aligned}
& e_{k}^{a} e_{\bar{i}}^{\bar{a}}=h_{k \bar{i}}, \quad e_{a}^{k} e_{\bar{a}}^{\bar{i}}=h^{\bar{i} k}, \quad h^{\bar{i} k} h_{k \bar{j}}=\delta_{\bar{j}}^{\bar{i}}, \quad h_{k \bar{j}} h^{\bar{j} l}=\delta_{k}^{l}, \\
& e_{k}^{a} e_{a}^{j}=\delta_{k}^{j}, \quad e_{a}^{k} e_{k}^{b}=\delta_{a}^{b}, \quad e_{\bar{k}}^{\bar{a}} e_{\bar{a}}^{\bar{j}}=\delta_{\bar{k}}^{\bar{j}}, \quad e_{\bar{a}}^{\bar{k}} e_{\bar{k}}^{\bar{b}}=\delta_{\bar{a}}^{\bar{b}} .
\end{aligned}
$$

\footnotetext{
${ }^{6}$ This is in contrast to Ref. [28, 27] where the Atiyah-Singer theorem on the manifolds involving extra curl-free torsion was considered.
} 
The nonzero components of the standard spin connections

$$
\Omega_{M, A B}=e_{A N}\left(\partial_{M} e_{B}^{N}+\Gamma_{M K}^{N} e_{B}^{K}\right)
$$

are

$$
\Omega_{j, \bar{b} a}=-\Omega_{j, a \bar{b}}=e_{p}^{b}\left(\partial_{j} e_{a}^{p}+\Gamma_{j k}^{p} e_{a}^{k}\right), \quad \Omega_{j, \bar{a} \bar{b}}=\frac{1}{2} e_{\bar{a}}^{\bar{s}} e_{\bar{b}}^{\bar{k}} C_{j \bar{s} \bar{k}}
$$

and complex conjugated $\bar{\Omega}_{\bar{j}, b \bar{a}}, \quad \bar{\Omega}_{\bar{j}, a b}$, with $C_{j \bar{k} \bar{s}}$ defined in (2.5).

When the torsion is present, one can define a generalized spin connection related to the generalized affine connection $\hat{\Gamma}_{M L}^{N}$ :

$$
\hat{\Omega}_{M, A B}=e_{A N}\left(\partial_{M} e_{B}^{N}+\hat{\Gamma}_{M K}^{N} e_{B}^{K}\right)=\Omega_{M, A B}-\frac{1}{2} e_{A}^{L} e_{B}^{K} C_{M L K} .
$$

The nonzero components of $\hat{\Omega}$ are

$$
\begin{aligned}
& \hat{\Omega}_{i, \bar{b} a}=-\hat{\Omega}_{i, a \bar{b}}=e_{k}^{b} \partial_{i} e_{a}^{k}+e_{\bar{b}}^{\bar{t}} e_{a}^{k} \hat{\Gamma}_{\bar{t}, i k}=e_{a}^{k} \partial_{k} e_{i}^{b}-e_{a}^{k} \partial_{i} e_{k}^{b}+e_{a}^{k} e_{\bar{b}}^{\bar{t}} e_{i}^{c} \partial_{k} e_{\bar{t}}^{\bar{c}}, \\
& \hat{\bar{\Omega}}_{\bar{i}, b \bar{a}}=\left(\hat{\Omega}_{i, \bar{b} a}\right)^{*}
\end{aligned}
$$

while the components $\hat{\Omega}_{j, \bar{a} \bar{b}}$ and $\hat{\Omega}_{\bar{j}, a b}$ vanish.

The vielbeins and the generalized spin connection satisfy the Maurer-Cartan structure equation

$$
d e_{A}+\hat{\Omega}_{A B} \wedge e_{B}=T_{A},
$$

where

$$
e_{A}=e_{A M} d x^{M}, \quad \hat{\Omega}_{A B}=\hat{\Omega}_{M, A B} d x^{M}, \quad T_{A}=\frac{1}{2} e_{A}^{M} C_{M N P} d x^{N} \wedge d x^{P} .
$$

The Maurer-Cartan equation for the standard torsion-free spin connection is

$$
d e_{A}+\Omega_{A B} \wedge e_{B}=0 .
$$

The equations (2.13) and (2.14) are equivalent, as can be checked using the relation (2.11). For the Hermitian metric (2.1), with the torsion defined in (2.5), these equations imply the identity

$$
\partial_{[k} e_{l]}^{a}-e_{\bar{a}}^{\bar{i}} e_{[k}^{d} \partial_{l]} e_{\bar{i}}^{\bar{d}}=\frac{1}{2} e_{\bar{a}}^{\bar{j}} C_{l k \bar{j}} \quad \text { (and c.c.) } .
$$

For Kähler manifolds, the metric (2.1) is derived from the Kähler potential,

$$
h_{j \bar{k}}(z, \bar{z})=\partial_{j} \partial_{\bar{k}} K(z, \bar{z}) \text {. }
$$

In this case

$$
\partial_{\bar{l}} h_{j \bar{k}}-\partial_{\bar{k}} h_{j \bar{l}}=\partial_{k} h_{j \bar{l}}-\partial_{j} h_{k \bar{l}}=0,
$$

and, as a result,

$$
C_{M N K}=0 \Rightarrow \hat{\Gamma}_{N K}^{M}=\Gamma_{N K}^{M}
$$


The only nonvanishing components of $\Gamma_{M, N P}$ in the Kähler case are:

$$
\Gamma_{\bar{m}, n p}=\partial_{n} h_{p \bar{m}}, \quad \Gamma_{m, \bar{n} \bar{p}}=\left(\Gamma_{\bar{m}, n p}\right)^{*}=\partial_{\bar{n}} h_{m \bar{p}} .
$$

The expressions for the non-vanishing components of the spin connections are also greatly simplified,

$$
\hat{\Omega}_{j, \bar{b} a}=\Omega_{j, \bar{b} a}=e_{\bar{b}}^{\bar{k}} \partial_{j} e_{\bar{k}}^{\bar{a}} \stackrel{\text { def }}{=} \omega_{j, \bar{b} a}, \quad \hat{\bar{\Omega}}_{\bar{j}, b \bar{a}}=\bar{\Omega}_{\bar{j}, b \bar{a}}=e_{b}^{k} \partial_{\bar{j}} e_{k}^{a} \stackrel{\text { def }}{=} \bar{\omega}_{\bar{j}, b \bar{a}} .
$$

In the Kähler case, the structure equation (2.15) acquires the form

$$
\partial_{[k} e_{l]}^{a}-e_{\bar{a}}^{\bar{i}} e_{[k}^{d} \partial_{l]} e_{\bar{i}}^{\bar{d}}=0 \quad \text { (and c.c.) }
$$

which is now a non-trivial constraint on the vielbeins $e_{l}^{a}, e_{\bar{l}}^{\bar{a}}$ ( equivalent to the constraint (2.17) on the metric). The only non-vanishing components of the Kähler Riemann tensor are

$$
\begin{aligned}
R_{j \bar{k}, l \bar{t}}= & \partial_{j} \partial_{\bar{k}} h_{l \bar{t}}-h_{p \bar{s}} \Gamma_{j l}^{p} \Gamma_{\bar{k} \bar{t}}^{\bar{s}}=\partial_{j} \partial_{\bar{k}} h_{l \bar{t}}-h^{\bar{s} n}\left(\partial_{j} h_{l \bar{s}}\right)\left(\partial_{\bar{k}} h_{n \bar{t}}\right) \\
& =e_{l}^{a} e_{\bar{t}}^{\bar{b}}\left(\partial_{j} \bar{\omega}_{\bar{k}, a \bar{b}}+\partial_{\bar{k}} \omega_{j, \bar{b} a}+\omega_{j, \bar{b} d} \bar{\omega}_{\bar{k}, a \bar{d}}-\omega_{j, \bar{d} a} \bar{\omega}_{\bar{k}, d \bar{b}}\right) .
\end{aligned}
$$

Finally, note the useful generic relations:

$$
\Omega_{i, \bar{a} b}=\omega_{i, \bar{a} b}+\frac{1}{2} e_{b}^{l} e_{\bar{a}}^{\bar{t}} C_{i l \bar{t}}, \quad \hat{\Omega}_{i, \bar{a} b}=\omega_{i, \bar{a} b}+e_{b}^{l} e_{\bar{a}}^{\bar{t}} C_{i l \bar{t}},
$$

where the expressions $\omega_{i, \bar{a} b}$ coincide by form with those defined in (2.20). Note that the objects $\omega_{i, \bar{a} b}$ can be given a geometric interpretation even in a non-Kähler case. They coincide with the appropriate components of a generalized spin connection associated with $\tilde{\Gamma}_{N K}^{M}=\Gamma_{N K}^{M}-\frac{1}{2} g^{M T} C_{T N K}$.

\section{$3 \mathcal{N}=2 \mathrm{SQM}$ model}

We formulate a general complex $\mathcal{N}=2, d=1 \mathrm{SQM}$ sigma model in terms of $2 n$ mutually conjugated chiral and anti-chiral superfields $Z^{j}\left(t_{L}, \theta\right), \bar{Z}^{\bar{j}}\left(t_{R}, \bar{\theta}\right)(j, \bar{j}=1, \cdots n)$,

$$
\bar{D} Z^{j}\left(t_{L}, \theta\right)=D \bar{Z}^{\bar{j}}\left(t_{R}, \bar{\theta}\right)=0,
$$

where

$$
D=\frac{\partial}{\partial \theta}-i \bar{\theta} \partial_{t}, \bar{D}=-\frac{\partial}{\partial \bar{\theta}}+i \theta \partial_{t}, \quad\{D, \bar{D}\}=2 i \partial_{t}, \quad t_{L}=t-i \theta \bar{\theta}, t_{R}=t+i \theta \bar{\theta}
$$

The basic superfields have the following component expansion

$$
Z^{j}=z^{j}+\sqrt{2} \theta \psi^{j}-i \theta \bar{\theta} \dot{z}^{j}, \quad \bar{Z}^{\bar{j}}=\bar{z}^{\bar{i}}-\sqrt{2} \bar{\theta} \bar{\psi}^{\bar{j}}+i \theta \bar{\theta} \dot{\bar{z}}^{\bar{j}} .
$$

The $\mathcal{N}=2$ transformation properties of the component fields are as follows:

$$
\begin{aligned}
& \delta z^{j}=-\sqrt{2} \epsilon \psi^{j}, \quad \delta \psi^{j}=\sqrt{2} i \bar{\epsilon}_{\bar{z}} \dot{z}^{j} \\
& \delta \bar{z}^{\bar{j}}=\sqrt{2} \bar{\epsilon} \bar{\psi}^{\bar{j}}, \quad \delta \bar{\psi}^{\bar{j}}=-\sqrt{2} i \epsilon \dot{\bar{z}}^{\bar{j}} .
\end{aligned}
$$


The superfield action we start with reads

$$
\begin{aligned}
& S=\int d t d^{2} \theta\left(\mathcal{L}_{\sigma}+\mathcal{L}_{\text {gauge }}\right), \\
& \mathcal{L}_{\sigma}=-\frac{1}{4} h_{i \bar{j}}(Z, \bar{Z}) D Z^{i} \bar{D} \bar{Z}^{\bar{j}}, \quad \mathcal{L}_{\text {gauge }}=W(Z, \bar{Z}) .
\end{aligned}
$$

Here, $h_{i \bar{j}}(Z, \bar{Z})$ and $W(Z, \bar{Z})$ are unconstrained functions of the superfields. In general [20], one can add to $\mathcal{L}$ the terms

$$
\sim \mathcal{B}_{i k}(Z, \bar{Z}) D Z^{i} D Z^{k}+\text { c.c. } .
$$

and also the terms involving higher antisymmetric even-dimensional tensors $\mathcal{B}_{i j k l}$, etc. These additional terms do not change the target space metric in the component action and affect only fermionic terms (introducing some extra non-zero components of the torsion). In this paper, we concentrate on the model where all these tensors vanish. The models with nonzero $\mathcal{B}$ were discussed in a recent paper [26].

The Kähler case corresponds to the choice

$$
h_{j \bar{k}}(Z, \bar{Z})=\partial_{j} \partial_{\bar{k}} K(Z, \bar{Z}),
$$

where the Kähler potential $K(Z, \bar{Z})$ is an arbitrary real function of the superfields 7 .

The component form of the full action is

$$
\begin{aligned}
S \equiv & \int d t\left(L_{\sigma}+L_{\text {gauge }}\right)=\int d t\left\{h_{j \bar{k}}\left[\dot{z}^{j} \dot{\bar{z}}^{\bar{k}}+\frac{i}{2}\left(\psi^{j} \dot{\bar{\psi}}^{\bar{k}}-\dot{\psi}^{j} \bar{\psi}^{\bar{k}}\right)\right]\right. \\
& -\frac{i}{2}\left[\left(2 \partial_{j} h_{t \bar{k}}-\partial_{t} h_{j \bar{k}}\right) \dot{z}^{t}-\left(2 \partial_{\bar{k}} h_{j \bar{t}}-\partial_{\bar{t}} h_{j \bar{k}}\right) \dot{\bar{z}}^{\bar{t}}\right] \psi^{j} \bar{\psi}^{\bar{k}}+\left(\partial_{t} \partial_{\bar{l}} h_{j \bar{k}}\right) \psi^{t} \psi^{j} \bar{\psi}^{\bar{l}} \bar{\psi}^{\bar{k}} \\
& \left.+2 \partial_{j} \partial_{\bar{k}} W \psi^{j} \bar{\psi}^{\bar{k}}-i\left(\partial_{j} W \dot{z}^{j}-\partial_{j} W \dot{\bar{z}}^{\bar{j}}\right)\right\}
\end{aligned}
$$

The geometric meaning of the different terms in the "sigma-model" part $L_{\sigma}$ of the Lagrangian in (3.8) can be clarified, if rewriting it in the following form

$$
L_{\sigma}=\frac{1}{2}\left[g_{M N} \dot{z}^{M} \dot{z}^{N}+i g_{M N} \psi^{M} \nabla \psi^{N}-\frac{1}{3 !} \partial_{P} C_{M N T} \psi^{P} \psi^{M} \psi^{N} \psi^{T}\right],
$$

where the metric $g_{M N}$ is written in (2.1), the covariant derivative $\nabla \psi^{N}$ was defined in (2.4) and the torsion tensor $C_{M N T}$ in (2.5), (2.6).

The Lagrangian $L_{\text {gauge }}$ (the last line in (3.8)) describes the interactions with the Abelian gauge field $A_{M}=\left(-i \partial_{j} W, i \partial_{\bar{j}} W\right) \equiv I_{M}^{N} \partial_{N} W$, the double derivative $F_{j \bar{k}}=$ $-F_{\bar{k} j}=2 i \partial_{j} \partial_{\bar{k}} W$ being the magnetic field strength. This Lagrangian can also be rewritten in a form analogous to (3.9)

$$
L_{\text {gauge }}=A_{M} \dot{Z}^{M}-\frac{i}{2} F_{M N} \psi^{M} \psi^{N} .
$$

\footnotetext{
${ }^{7}$ For $\mathbb{C P}^{1}$ and with the restriction $K=W$, this SQM model was earlier considered at the classical (component and superfield) and quantum levels in Refs. 29, 30, 31.
} 
The prepotential $W(Z, \bar{Z})$ is an arbitrary function. A particularly clever choice is $W \propto \ln \operatorname{det} h$ (see Eq.(5.1) below). The corresponding bundle $\left(-i \partial_{j} W, i \partial_{j} W\right)$ is called canonical by mathematicians.

The fermion variables $\psi^{j}, \bar{\psi}^{\bar{k}}$ are not canonically conjugated, their Poisson bracket being equal to $-i h^{\bar{k} j}$. It is convenient to introduce the canonically conjugated fermionic fields with the tangent space indices,

$$
\psi^{a}=e_{j}^{a} \psi^{j}, \quad \bar{\psi}^{\bar{b}}=e_{\bar{k}}^{\bar{b}} \bar{\psi}^{\bar{k}}
$$

such that all the variables have the canonical Poisson brackets,

$$
\left\{z^{j}, P_{k}\right\}_{P B}=\delta_{k}^{j}, \quad\left\{\bar{z}^{\bar{j}}, P_{\bar{k}}\right\}_{P B}=\delta_{\bar{k}}^{\bar{j}}, \quad\left\{\psi^{a}, \bar{\psi}^{\bar{b}}\right\}_{P B}=-i \delta^{a \bar{b}} .
$$

Then, using the invariance of the Lagrangian in (3.8) under the transformations (3.4) (modulo a total time derivative), it is easy to compute the corresponding canonical supercharges

$$
\begin{aligned}
& Q=\sqrt{2}\left[\Pi_{k} e_{a}^{k} \psi^{a}-i \psi^{b} \psi^{d} \bar{\psi}^{\bar{a}}\left(e_{b}^{k} \partial_{[k} e_{l]}^{a} e_{d}^{l}\right)\right] \\
& \bar{Q}=\sqrt{2}\left[\bar{\Pi}_{\bar{k}} e_{\bar{a}}^{\bar{k}} \bar{\psi}^{\bar{a}}-i \bar{\psi}^{\bar{c}} \bar{\psi}^{\bar{a}} \psi^{d}\left(e_{\bar{c}}^{\bar{k}} \partial_{[\bar{k}} e_{\bar{l}]}^{\bar{d}} e_{\bar{a}}^{\bar{l}}\right)\right]
\end{aligned}
$$

where

$$
\Pi_{k}=P_{k}+i \partial_{k} W, \quad \bar{\Pi}_{\bar{k}}=P_{\bar{k}}-i \partial_{\bar{k}} W
$$

and $P_{k}, P_{\bar{k}}$ are the canonical momenta.

Using the definitions (2.10), (2.5) and the relations (2.15), (2.23), these supercharges can be brought in a more suggestive geometric form

$$
Q=\sqrt{2}\left[\Pi_{k}-i \bar{\psi}^{\bar{a}} \psi^{b} \Omega_{k, \bar{a} b}\right] e_{c}^{k} \psi^{c}, \quad \bar{Q}=\sqrt{2} e_{\bar{c}}^{\bar{k}} \bar{\psi}^{\bar{c}}\left[\bar{\Pi}_{\bar{k}}+i \bar{\psi}^{\bar{a}} \psi^{d} \bar{\Omega}_{\bar{k}, d \bar{a}}\right] .
$$

It should be pointed out that the 3-fermionic terms in these supercharges contain the spin connections $\Omega_{k, \bar{a} b}, \bar{\Omega}_{\bar{k}, d \bar{a}}$ corresponding to the standard symmetric Christoffel symbols $\Gamma_{M K}^{N}$ and defined by the relations (2.10), (2.23). 8

Using (3.12), it is easy to find

$$
\{Q, \bar{Q}\}_{P B}=-2 i H_{c l},\{Q, Q\}_{P B}=\{\bar{Q}, \bar{Q}\}_{P B}=\left[Q, H_{c l}\right]_{P B}=\left[\bar{Q}, H_{c l}\right]_{P B}=0 .
$$

The canonical classical Hamiltonian $H_{c l}$ can be represented in the following compact form:

$$
\begin{aligned}
& H_{c l}=h^{\bar{k} j}\left(\Pi_{j}-i \hat{\Omega}_{j, \bar{b} a} \bar{\psi}^{\bar{b}} \psi^{a}\right)\left(\bar{\Pi}_{\bar{k}}+i \hat{\bar{\Omega}}_{\bar{k}, c \bar{d}} \bar{\psi}^{\bar{d}} \psi^{c}\right) \\
& \quad-2 e_{a}^{j} e_{\bar{b}}^{\bar{k}} \partial_{j} \partial_{\bar{k}} W \psi^{a} \bar{\psi}^{\bar{b}}-e_{a}^{t} e_{c}^{j} e_{\bar{b}}^{\bar{l}} e_{\bar{d}}^{\bar{k}}\left(\partial_{t} \partial_{\bar{l}} h_{j \bar{k}}\right) \psi^{a} \psi^{c} \bar{\psi}^{\bar{b}} \bar{\psi}^{\bar{d}}
\end{aligned}
$$

It is interesting to note that, in the generic case, the spin connections entering the supercharges (3.15) and the classical hamiltonian (3.17) do not coincide with each other: they differ by a term proportional to the torsion.

\footnotetext{
${ }^{8} \mathrm{On}$ the other hand, the supercharges involve only the holomorphic components of the spin connections and do not depend on non-holomorphic components $\Omega_{k, \bar{a} \bar{b}}, \Omega_{\bar{k}, a b}$. See also Eq.(3.35) below.
} 
In the Kähler case, when the torsion is vanishing, the situation simplifies. Both the supercharges and the Hamiltonian are expressed through the same connections (2.20). In addition, the last four-fermionic term in (3.17) vanishes.

We thus obtain

$$
Q^{\mathrm{Kähl}}=\sqrt{2}\left[\Pi_{k}-i \bar{\psi}^{\bar{a}} \psi^{b} \omega_{k, \bar{a} b}\right] e_{c}^{k} \psi^{c}, \quad \bar{Q}^{\mathrm{Kähl}}=\sqrt{2} e_{\bar{c}}^{\bar{k}} \bar{\psi}^{\bar{c}}\left[\bar{\Pi}_{\bar{k}}+i \bar{\psi}^{\bar{a}} \psi^{d} \bar{\omega}_{\bar{k}, d \bar{a}}\right]
$$

and

$$
H_{c l}^{\mathrm{Kähl}}=h^{\bar{k} j}\left(\Pi_{j}-i \omega_{j, \bar{b} a} \bar{\psi}^{\bar{b}} \psi^{a}\right)\left(\bar{\Pi}_{\bar{k}}+i \bar{\omega}_{\bar{k}, c \bar{d}} \bar{\psi}^{\bar{d}} \psi^{c}\right)-2 e_{a}^{j} e_{\bar{b}}^{\bar{k}} \partial_{j} \partial_{\bar{k}} W \psi^{a} \bar{\psi}^{\bar{b}} .
$$

The expression for the Lagrangian also simplifies a lot in the Kähler case. The four-fermionic term in (3.8), (3.9) vanishes. The remaining terms in $L_{\sigma}$ can be presented as

$$
L_{\sigma}^{\mathrm{Kähl}}=h_{j \bar{k}} \dot{z}^{j} \dot{\bar{z}}^{k}+\frac{i}{2}\left(\psi^{a} \dot{\bar{\psi}}^{\bar{a}}-\dot{\psi}^{a} \bar{\psi}^{a}\right)+i\left(\dot{\bar{z}}^{\bar{k}} \bar{\omega}_{\bar{k}, a \bar{b}}-\dot{z}^{k} \omega_{k, \bar{b} a}\right) \psi^{a} \bar{\psi}^{\bar{b}} .
$$

Let us now turn to quantum theory. The Poisson brackets (3.12) are replaced by the (anti)commutators:

$$
\left[z^{j}, P_{k}\right]=i \delta_{k}^{j},\left[\bar{z}^{\bar{j}}, P_{\bar{k}}\right]=i \delta_{\bar{k}}^{\bar{j}},\left\{\psi^{a}, \bar{\psi}^{\bar{b}}\right\}=\delta^{a \bar{b}} .
$$

As is well known, there exist, generically, many different quantum theories corresponding to a given classical one, due to ordering ambiguities. To make a selection, we require that the supersymmetry algebra (3.16) remains intact at the quantum level and that $Q_{q u}$ and $\bar{Q}_{q u}$ are Hermitian conjugate to each other. As was noticed in [32, these two requirements can be simultaneously fulfilled only provided that the classical expressions for the supercharges are Weyl-ordered in the quantum case. The correct expression for the quantum Hamiltonian is obtained as the anticommutator of the Weyl-ordered $Q_{q u}$ and $\bar{Q}_{q u}$. Note that this correct quantum Hamiltonian does not coincide with the operator obtained through Weyl-ordering of the classical Hamiltonian defined by the relations (3.16).

We thus obtain

$$
\begin{aligned}
Q^{\mathrm{flat}} & =\frac{1}{\sqrt{2}}\left[\left\{\Pi_{k}, e_{a}^{k}\right\} \psi^{a}+i\left\{\psi^{b} \psi^{d}, \bar{\psi}^{\bar{a}}\right\} e_{b}^{k} \Omega_{k, \bar{a} d}\right], \\
\bar{Q}^{\mathrm{flat}} & =\frac{1}{\sqrt{2}}\left[\left\{\bar{\Pi}_{\bar{k}}, e_{\bar{a}}^{\bar{k}}\right\} \bar{\psi}^{\bar{a}}+i\left\{\bar{\psi}^{\bar{c}} \bar{\psi}^{\bar{a}}, \psi^{d}\right\} e_{\bar{c}}^{\bar{k}} \bar{\Omega}_{\bar{k}, d \bar{a}}\right] .
\end{aligned}
$$

These Weyl-ordered supercharges were dubbed "flat" because they act on the wave functions normalized by the condition 32

$$
\int \prod_{k} d z^{k} d \bar{z}^{\bar{k}} \prod_{a} d \psi^{a} d \bar{\psi}^{\bar{a}} \exp \left\{\bar{\psi}^{\bar{a}} \psi^{a}\right\} \bar{\Psi}\left(z^{k}, \bar{z}^{\bar{k}}, \bar{\psi}^{\bar{a}}\right) \Psi\left(\bar{z}^{\bar{k}}, z^{k}, \psi^{a}\right)=1
$$

with the flat Hilbert space measure. In particular, it is straightforward to see that the Weyl-ordered supercharges $Q$ and $\bar{Q}$ are Hermitian-conjugate to each other with respect to such flat inner product 9 .

${ }^{9}$ The same concerns the fermion operators $\psi^{a}$ and $\bar{\psi}^{\bar{a}}=\frac{\partial}{\partial \psi^{a}}$. They are Hermitian-conjugated with a particular Berezin integration measure in (3.23), (3.24) involving the factor $\exp \left\{\bar{\psi}^{\bar{a}} \psi^{a}\right\}$. 
It is more natural, however, to deal with the covariant supercharges $Q^{\text {cov }}, \bar{Q}^{\text {cov }}$ which act on the Hilbert space in which the inner product is defined with the covariant integration measure

$$
\int \prod_{k} d z^{k} d \bar{z}^{\bar{k}} \operatorname{det} h \prod_{a} d \psi^{a} d \bar{\psi}^{\bar{a}} \exp \left\{\bar{\psi}^{\bar{a}} \psi^{a}\right\} \bar{\Psi}\left(z^{k}, \bar{z}^{\bar{k}}, \bar{\psi}^{\bar{a}}\right) \Psi\left(\bar{z}^{\bar{k}}, z^{k}, \psi^{a}\right)=1
$$

(note that $\operatorname{det} h \propto \sqrt{\operatorname{det} g}$ ). They are related to the flat supercharges by a similarity transformation

$$
\left(Q^{\mathrm{cov}}, \bar{Q}^{\mathrm{cov}}\right)=(\operatorname{det} h)^{-1 / 2}\left(Q^{\mathrm{flat}}, \bar{Q}^{\mathrm{flat}}\right)(\operatorname{det} h)^{1 / 2}
$$

which yields the expressions:

$$
\begin{aligned}
Q^{\mathrm{cov}} & =\sqrt{2} \psi^{c} e_{c}^{k}\left[\Pi_{k}-\frac{i}{2} \partial_{k}(\ln \operatorname{det} \bar{e})+i \psi^{b} \bar{\psi}^{\bar{a}} \Omega_{k, \bar{a} b}\right] \\
\bar{Q}^{\mathrm{cov}} & =\sqrt{2} \bar{\psi}^{\bar{c}} e_{\bar{c}}^{\bar{k}}\left[\bar{\Pi}_{\bar{k}}-\frac{i}{2} \partial_{\bar{k}}(\ln \operatorname{det} e)+i \bar{\psi}^{\bar{b}} \psi^{a} \bar{\Omega}_{\bar{k}, a \bar{b}}\right] .
\end{aligned}
$$

Here,

$$
\Pi_{k}=-i\left(\frac{\partial}{\partial z^{k}}-\partial_{k} W\right), \quad \bar{\Pi}_{\bar{k}}=-i\left(\frac{\partial}{\partial \bar{z}^{\bar{k}}}+\partial_{\bar{k}} W\right) .
$$

The supercharges obey the relations of the $\mathcal{N}=2, d=1$ Poincaré superalgebra $\left\{Q^{\mathrm{cov}}, \bar{Q}^{\mathrm{cov}}\right\}=2 H_{q u}^{\mathrm{cov}},\left\{Q^{\mathrm{cov}}, Q^{\mathrm{cov}}\right\}=\left\{\bar{Q}^{\mathrm{cov}}, \bar{Q}^{\mathrm{cov}}\right\}=\left[Q^{\mathrm{cov}}, H_{q u}^{\mathrm{cov}}\right]=\left[\bar{Q}^{\mathrm{cov}}, H_{q u}^{\mathrm{cov}}\right]=0$.

The expression for the quantum Hamiltonian $H_{q u}^{\text {cov }}$ can be obtained in two ways: (i) By directly calculating the anticommutator of quantum supercharges (3.26) or (ii) by Weyl-ordering the Grönewold-Moyal bracket [33, 34] of the classical supercharges (3.15) and performing then the similarity transformation, like in (3.25).

We obtain

$$
\begin{aligned}
H_{q u}^{\mathrm{cov}}= & -\frac{1}{2} \triangle^{\mathrm{cov}}+\frac{1}{8}\left(R-\frac{1}{2} h^{\bar{k} j} h^{\bar{l} t} h^{\bar{i} n} C_{j t \bar{i}} C_{\bar{k} \bar{l} n}\right) \\
& -2\left\langle\psi^{a} \bar{\psi}^{\bar{b}}\right\rangle e_{a}^{k} e_{\bar{b}}^{\bar{l}} \partial_{k} \partial_{\bar{l}} W-\left\langle\psi^{a} \psi^{c} \bar{\psi}^{\bar{b}} \bar{\psi}^{\bar{d}}\right\rangle e_{a}^{t} e_{c}^{j} e_{\bar{b}}^{\bar{l}} e_{\bar{d}}^{\bar{k}}\left(\partial_{t} \partial_{\bar{l}} h_{j \bar{k}}\right) .
\end{aligned}
$$

Here, $\langle\ldots\rangle$ denotes the Weyl-ordered products of fermions, $R$ is the standard scalar curvature of the metric $h_{j \bar{k}}$, and $\triangle^{\text {cov }}$ is the covariant Laplacian calculated with the "hatted" affine connections in (2.7) and including also the (hatted) spin connections,

$$
-\triangle^{\mathrm{cov}}=h^{\bar{k} j}\left(\mathcal{P}_{j} \overline{\mathcal{P}}_{\bar{k}}+i \hat{\Gamma}_{j \bar{k}}^{\bar{q}} \overline{\mathcal{P}}_{\bar{q}}+\overline{\mathcal{P}}_{\bar{k}} \mathcal{P}_{j}+i \hat{\Gamma}_{\bar{k} j}^{s} \mathcal{P}_{s}\right)
$$

where $\mathcal{P}_{j}=\Pi_{j}+i \hat{\Omega}_{j, \bar{b} a}\left\langle\psi^{a} \bar{\psi}^{\bar{b}}\right\rangle$ and $\overline{\mathcal{P}}_{\bar{k}}=\bar{\Pi}_{\bar{k}}-i \hat{\bar{\Omega}}_{\bar{k}, a \bar{b}}\left\langle\psi^{a} \bar{\psi}^{\bar{b}}\right\rangle$. Note that the scalar curvature $R$ is related to its "hatted" counterpart $\hat{R}$ associated with the non-symmetric affine connection $\hat{\Gamma}$ by the simple formula

$$
\hat{R}=R-\frac{1}{4} C_{M N P} C^{M N P}=R-\frac{3}{2} h^{\bar{k} j} h^{\bar{l} t} h^{\overline{i n}} C_{j t \bar{i}} C_{\bar{k} \bar{l} n} .
$$


In the Kähler case, the expression for the quantum Hamiltonian greatly simplifies:

$$
H_{\text {Kăhl }}^{\text {cov }}=-\frac{1}{2} \triangle^{\text {cov }}+\frac{R}{8}-2\left\langle\psi^{a} \bar{\psi}^{\bar{b}}\right\rangle e_{a}^{k} e_{\bar{b}}^{\bar{l}} \partial_{k} \partial_{\bar{l}} W,
$$

where now $-\triangle^{\text {cov }}=h^{\bar{k} j}\left(\mathcal{P}_{j} \overline{\mathcal{P}}_{\bar{k}}+\overline{\mathcal{P}}_{\bar{k}} \mathcal{P}_{j}\right)$ and $\hat{\Omega}, \hat{\bar{\Omega}}$ are reduced to $\Omega, \bar{\Omega}=\omega, \bar{\omega}$ according to the relations (2.20).

An important remark is to the point here. The Lagrangian (3.20) can also be expressed through real variables,

$$
L=\frac{g_{M N}}{2}\left[\dot{z}^{M} \dot{z}^{N}+i \psi^{M} \nabla \psi^{N}\right]=\frac{1}{2}\left[g_{M N} \dot{z}^{M} \dot{z}^{N}+i \psi^{A}\left(\dot{\psi}^{A}+\Omega_{M, A B} \dot{z}^{M} \psi^{B}\right)\right] .
$$

This Lagrangian is well known [14, 15]. It can be (and was) also considered for a generic (not necessarily complex) manifold. In a generic case, it is manifestly invariant only under $\mathcal{N}=1$ supersymmetry transformations (with a real Grassmann parameter). The corresponding Nöther supercharge is

$$
\mathcal{Q} \sim \psi^{A} e_{A}^{M}\left[P_{M}-\frac{i}{2} \Omega_{M, B C} \psi^{B} \psi^{C}\right] .
$$

The covariant quantum supercharge (obtained by Weyl-ordering of the classical supercharge and taking a correct account of the measure factor as in (3.25)) is given by the same expression, where now $\left\{\psi^{A}, \psi^{B}\right\}=\delta^{A B}$. It can be interpreted as the Dirac operator. By construction, it is Hermitian with the Hilbert space metric including the factor $\sqrt{\operatorname{det} g}$.

The corresponding quantum Hamiltonian [6, 7] coincides, up to a proper similarity transformation, with (3.30) rewritten in real notations.

As was mentioned in the Introduction (and we will return to the discussion of this issue in Sect. 6), for an even-dimensional manifold, the second real supercharge

$$
\tilde{\mathcal{Q}}=2^{D / 2} \mathcal{Q} \prod_{A=1}^{D} \psi^{A}
$$

associated with $\not \mathcal{D} \gamma^{D+1}$ can also be defined. However, for $D \geq 4$, this second supercharge has nothing to do with the supercharges (3.26).

To recapitulate:

- For any even-dimensional manifold, the system (3.31) admits two real quantum supercharges (3.32) and (3.33).

- For any manifold with Hermitian metric (2.1), a generically different system (3.8) involves a different pair of supercharges (3.26). We will show in Sect. 5 that these supercharges can be interpreted as an exterior holomorphic derivative and its complex conjugate.

- We will also show in Sect. 5 that, in the Kähler case, the imaginary and real parts of the supercharge $Q$ in (3.26) can be interpreted as the Dirac operator $\not D$ and the operator $S$ defined in Eq. (1.4). 
- Thus, for Kähler manifolds, when the Lagrangians (3.8) and (3.31) coincide, two different $\mathcal{N}=2$ supersymmetry structures $\left(\not^{2} ; \not{D}, \not{D} \gamma^{D+1}\right)$ and $\left(\not^{2} ; \not{D}, S\right)$ are possible. Note that this does not imply an extended $\mathcal{N}=4$ supersymmetry because the anticommutator $\left\{S, \not{D} \gamma^{D+1}\right\}$ does not vanish.

- Note, however, that, for hyper-Kähler manifolds where three different complex structures are present, one can construct three different new supercharges

$$
S^{(f)}=I_{N}^{M(f)} \gamma^{N} \mathcal{D}_{M}, \quad f=1,2,3,
$$

such that the Lagrangian (3.20), (3.31) enjoys an $\mathcal{N}=4$ supersymmetry $21] 10$.

- There exists also an $\mathcal{N}=4$ completion of the system (3.20) for any Kähler manifold, as will be discussed in Sect. 4 .

- For non-Kähler manifolds, the sum $\mathcal{Q}=\bar{Q}+Q$ can also be interpreted as a Dirac operator, but with some extra torsions. Indeed, one can show that

$$
\mathcal{Q}=\sqrt{2} \psi^{M}\left[\Pi_{M}-\frac{i}{2} \Omega_{M, B C} \psi^{B} \psi^{C}+\frac{i}{12} C_{M K L} \psi^{K} \psi^{L}\right],
$$

which may be interpreted as a torsionful Dirac operator where the torsion tensor involves an extra factor $1 / 3$ compared to the Bismut connection [37].

\subsection{Examples}

Here we consider two examples of SQM on complex manifolds.

1. $\mathbb{C P}^{n}$ model. This is a Kähler manifold, so the torsion (2.5) vanishes and many formulas look simpler. The corresponding Kähler potential is

$$
K=\ln (1+z \bar{z}), \quad z \bar{z} \equiv z^{j} \bar{z}^{\bar{j}} .
$$

We choose

$$
W=-\frac{q}{2} K=-\frac{q}{2} \ln (1+z \bar{z}) .
$$

As we will see in Sect. 5, the quantum problem is consistent when the constant $q$ is integer for odd $n$ and half-integer for even $n$.

The metric is given by the well known Fubini-Study expressions:

$$
\begin{aligned}
& h_{j \bar{k}}=\partial_{j} \partial_{\bar{k}} \ln (1+z \bar{z})=\frac{1}{1+z \bar{z}}\left(\delta_{j \bar{k}}-\frac{z^{k} \bar{z}^{\bar{j}}}{1+z \bar{z}}\right), \\
& h^{\bar{k} j}=(1+z \bar{z})\left(\delta^{\bar{k} j}+z^{j} \bar{z}^{\bar{k}}\right) .
\end{aligned}
$$

\footnotetext{
${ }^{10}$ This $\mathcal{N}=4$ supersymmetry holds also for more general class of sigma models associated with the so called HKT (hyper-Kähler with torsion) manifolds [35]. Their off-shell superfield description was given in a recent paper [36].
} 
Note the specific for $\mathbb{C P}^{n}$ relation

$$
K=-\frac{1}{n+1} \ln \operatorname{det} h
$$

We choose the vielbeins in the form [21]:

$$
\begin{aligned}
& e_{l}^{a}=\frac{1}{\sqrt{1+z \bar{z}}}\left(\delta_{l}^{a}-\frac{z^{a} \bar{z}^{\bar{l}}}{\sqrt{1+z \bar{z}}(1+\sqrt{1+z \bar{z}})}\right), \\
& e_{b}^{l}=\sqrt{1+z \bar{z}}\left(\delta_{b}^{l}+\frac{z^{l} \bar{z}^{\bar{b}}}{1+\sqrt{1+z \bar{z}}}\right), \\
& e_{\bar{a}}^{\bar{l}}=\sqrt{1+z \bar{z}}\left(\delta_{\bar{a}}^{\bar{l}}+\frac{z^{a} \bar{z}^{\bar{l}}}{1+\sqrt{1+z \bar{z}}}\right), \\
& e_{\bar{l}}^{\bar{a}}=\frac{1}{\sqrt{1+z \bar{z}}}\left(\delta_{\bar{l}}^{\bar{a}}-\frac{z^{l} \bar{z}^{\bar{a}}}{\sqrt{1+z \bar{z}}(1+\sqrt{1+z \bar{z}})}\right) .
\end{aligned}
$$

The supercharges (3.26) in this special case look as follows

$$
Q^{\mathrm{cov}}=\sqrt{2} \psi^{c} e_{c}^{k}\left[\tilde{\Pi}_{k}+i \psi^{b} \bar{\psi}^{\bar{a}} \omega_{k, \bar{a} b}\right], \quad \bar{Q}^{\mathrm{cov}}=\sqrt{2} \bar{\psi}^{\bar{c}} e_{\bar{c}}^{\bar{k}}\left[\overline{\tilde{\Pi}}_{\bar{k}}+i \bar{\psi}^{\bar{a}} \psi^{b} \bar{\omega}_{\bar{k}, b \bar{a}}\right],
$$

where

$$
\begin{aligned}
& \tilde{\Pi}_{k}=\frac{1}{i}\left[\frac{\partial}{\partial z^{k}}+\frac{1}{2}\left(q-\frac{n+1}{2}\right) \frac{\bar{z}^{\bar{k}}}{1+z \bar{z}}\right], \\
& \hat{\bar{\Pi}}_{\bar{k}}=\frac{1}{i}\left[\frac{\partial}{\partial \bar{z}^{\bar{k}}}-\frac{1}{2}\left(q+\frac{n+1}{2}\right) \frac{z^{k}}{1+z \bar{z}}\right]
\end{aligned}
$$

and $\omega_{k, \bar{a} b}, \bar{\omega}_{\bar{k}, b \bar{a}}$ were defined in Eq.(2.20) $)$.

2. $S^{4}$ model. As a second example, we consider a 4-dimensional conformally flat manifold with the metric

$$
d s^{2}=\frac{2 d z^{j} d \bar{z}^{\bar{j}}}{f^{2}}, \quad j=1,2 .
$$

When $f=1+z \bar{z}$, this is the metric of $S^{4}$ or rather $S^{4} \backslash\{\cdot\}$ (the metric (3.43) being singular in infinity). Under a natural choice of vielbeins, $\operatorname{det} e=\operatorname{det} \bar{e}=1 / f^{2}$ and the non-zero components of the spin connection $\Omega_{k, \bar{a} b}$ are

$$
\Omega_{1, \overline{1} 1}=\Omega_{2, \overline{2} 1}=-\partial_{1} \ln f, \quad \Omega_{1, \overline{1} 2}=\Omega_{2, \overline{2} 2}=-\partial_{2} \ln f .
$$

This is not a Kähler manifold. Taking the general expression (3.26) for the supercharges, we derive for $W=0$,

$$
\mathcal{Q}=-i \sqrt{2} \psi^{a}\left[f \partial_{a}-\left(\partial_{a} f\right)\right]-i \sqrt{2} \psi^{1} \psi^{2}\left[\left(\partial_{2} f\right) \bar{\psi}^{1}-\left(\partial_{1} f\right) \bar{\psi}^{2}\right]
$$


An $\mathcal{N}=4$ SQM model describing the motion over any conformally flat 4-dimensional manifold with the metric (3.43) with or without background gauge field was constructed in [38] based on the action given in [39] (see also [40]). In the case when the gauge field is absent, the flat (in the Hilbert space sense, as discussed above) supercharges have the form

$$
\begin{aligned}
Q_{\alpha} & =f\left(\sigma_{\mu} \bar{\psi}\right)_{\alpha} P_{\mu}-i\left(\partial_{\mu} f\right) \psi_{\gamma} \bar{\psi}^{\gamma}\left(\sigma_{\mu} \bar{\psi}\right)^{\alpha} \\
\bar{Q}^{\alpha} & =\left(\psi \sigma_{\mu}^{\dagger}\right)^{\alpha} P_{\mu} f+i\left(\partial_{\mu} f\right)\left(\psi \sigma_{\mu}^{\dagger}\right)^{\alpha} \psi_{\gamma} \bar{\psi}^{\gamma}
\end{aligned}
$$

where $\sigma_{\mu}=(i, \vec{\sigma}), \quad \sigma_{\mu}^{\dagger}=(-i, \vec{\sigma})$.

It is straightforward to see that, after performing the similarity transformation (3.25), the supercharge (3.45) coincides with $\bar{Q}^{1}$ in (3.46) under the identification

$$
z^{1}=\frac{x_{3}+i x_{4}}{\sqrt{2}}, \quad z^{2}=\frac{x_{1}-i x_{2}}{\sqrt{2}}
$$

or with $\bar{Q}^{2}$, under the identification

$$
z^{1}=\frac{x_{1}+i x_{2}}{\sqrt{2}}, \quad z^{2}=\frac{i x_{4}-x_{3}}{\sqrt{2}}
$$

These two possibilities reflect the presence of two different $\mathcal{N}=2$ Poincaré superalgebras in the $\mathcal{N}=4$ superalgebra.

In the case of $S^{4} \backslash\{\cdot\}$, the spectrum of the model was recently analyzed in [23]. In spite of the singularity, which could ruin the supersymmetry [24], this does not happen in this case. The spectrum is supersymmetric, involving 3 bosonic zero modes.

\section{Completion to Kähler $\mathcal{N}=4 \mathrm{SQM}$ model}

Our starting point is the $\mathcal{N}=2$ SQM model with the superfield Lagrangian $\mathcal{L}_{\sigma}$ in (3.5) involving the Kähler metric (3.7). We do not add the gauge part $\mathcal{L}_{\text {gauge }}$. So we choose

$$
h_{j \bar{k}}(Z, \bar{Z})=\partial_{j} \partial_{\bar{k}} K(Z, \bar{Z}), \quad W=0
$$

in (3.5). The corresponding component Lagrangian was written in (3.20).

Using the chirality properties of $Z^{j}, \bar{Z}^{\bar{k}}$ and the algebra of $\mathcal{N}=2$ spinor derivatives, it will be convenient to rewrite the corresponding superfield Lagrangian in the following three equivalent (they coincide up to a total time derivative) forms:

$$
\begin{aligned}
\mathcal{L}^{K} & =-\frac{1}{4} \partial_{j} \partial_{\bar{k}} K(Z, \bar{Z}) D Z^{j} \bar{D} \bar{Z}^{\bar{k}} \simeq-\frac{i}{2} \dot{Z}^{j} \partial_{j} K \\
& \simeq \frac{i}{2} \dot{\bar{Z}}^{\bar{k}} \partial_{\bar{k}} K \simeq \frac{i}{4}\left(\dot{\bar{Z}}^{\bar{k}} \partial_{\bar{k}} K-\dot{Z}^{j} \partial_{j} K\right) .
\end{aligned}
$$

Now consider an extended Lagrangian

$$
\tilde{\mathcal{L}}^{K}=\mathcal{L}^{K}+\frac{1}{4} h_{j \bar{k}} \Phi^{j} \bar{\Phi}^{\bar{k}}
$$


where $\Phi^{j}, \bar{\Phi}^{\bar{k}}$ are chiral and anti-chiral fermionic $\mathcal{N}=2(0+1)$-dimensional superfields, $\bar{D} \Phi^{j}=D \bar{\Phi}^{\bar{k}}=0$. It is straightforward to check that (4.3) is invariant, modulo a total derivative, under the following extra $\mathcal{N}=2$ supersymmetry transformations:

$$
\delta Z^{j}=-\zeta \Phi^{j}, \quad \delta \bar{Z}^{\bar{k}}=\bar{\zeta} \bar{\Phi}^{\bar{k}}, \quad \delta \Phi^{j}=2 i \bar{\zeta} \dot{Z}^{j}, \quad \delta \bar{\Phi}^{\bar{k}}=-2 i \zeta \dot{\bar{Z}}^{\bar{k}} .
$$

These variations form the same algebra with respect to Lie brackets as the variations (3.4) corresponding to the manifest world-line $\mathcal{N}=2$ supersymmetry. Thus, they extend the latter to off-shell $(0+1)$-dimensional $\mathcal{N}=4$ supersymmetry.

The superfields $\Phi^{j}, \bar{\Phi}^{\bar{k}}$ have the following $\theta$ expansions

$$
\Phi^{j}=\sqrt{2} \chi^{j}+\theta d^{j}-i \sqrt{2} \theta \bar{\theta} \dot{\chi}^{j}, \quad \bar{\Phi}^{\bar{k}}=\sqrt{2} \bar{\chi}^{\bar{k}}+\bar{\theta} \bar{d}^{\bar{k}}+i \sqrt{2} \theta \bar{\theta} \dot{\bar{\chi}}^{\bar{k}} .
$$

We observe that they contain no new bosonic fields of physical dimension, only the auxiliary bosonic fields $d^{j}, \bar{d}^{\bar{k}}$ as well as the extra physical fermionic fields $\chi^{j}, \bar{\chi}^{\bar{k}}$. Thus, in this model we deal with $n$ off-shell $\mathcal{N}=4$ supermultiplets $(\mathbf{2}, \mathbf{4}, \mathbf{2})$, the subsequent numerals standing, respectively, for the numbers of the physical bosonic, physical fermionic and auxiliary bosonic field 11 . The manifest $\mathcal{N}=2$ supersymmetry acts on the component fields in (4.5) as

$$
\delta \chi^{j}=-\frac{1}{\sqrt{2}} \epsilon d^{j}, \quad \delta d^{j}=2 \sqrt{2} i \bar{\epsilon} \dot{\chi}^{j}, \quad \delta \bar{\chi}^{\bar{k}}=-\frac{1}{\sqrt{2}} \bar{\epsilon} \bar{d}^{\bar{k}}, \quad \delta \bar{d}^{\bar{k}}=2 \sqrt{2} i \epsilon \dot{\bar{\chi}}^{\bar{k}} .
$$

The second supersymmetry transformations (4.4) has the following realization in components:

$$
\begin{aligned}
& \delta z^{j}=-\sqrt{2} \zeta \chi^{j}, \delta \psi^{j}=\frac{1}{\sqrt{2}} \zeta d^{j}, \delta \chi^{j}=\sqrt{2} i \bar{\zeta}^{j}, \delta d^{j}=-2 \sqrt{2} i \bar{\zeta}^{j} \dot{\psi}^{j}, \\
& \delta \bar{z}^{\bar{k}}=\sqrt{2} \bar{\zeta} \bar{\chi}^{\bar{k}}, \delta \bar{\psi}^{\bar{k}}=\frac{1}{\sqrt{2}} \bar{\zeta} \bar{d}^{\bar{k}}, \delta \bar{\chi}^{\bar{k}}=-\sqrt{2} i \zeta \dot{\bar{z}}^{\bar{k}}, \delta \bar{d}^{\bar{k}}=-2 \sqrt{2} i \zeta \dot{\bar{\psi}}^{\bar{k}} .
\end{aligned}
$$

After going to the component fields in the action corresponding to the modified superfield Lagrangian (4.3) and eliminating the auxiliary fields $d^{j}, \bar{d}^{\bar{k}}$ by their equations of motion,

$$
d^{j}=2 h^{\bar{p} j} \partial_{l} h_{t \bar{p}} \chi^{t} \psi^{l}, \quad \bar{d}^{\bar{k}}=2 h^{\bar{k} p} \partial_{\bar{l}} h_{p \bar{j}} \bar{\psi}^{\bar{l}} \bar{\chi}^{\bar{j}}
$$

the contribution of the second term in (4.3) to the total component Lagrangian reads:

$$
\Delta L=\frac{i}{2} h_{j \bar{k}}\left(\chi^{j} \nabla \bar{\chi}^{\bar{k}}-\nabla \chi^{j} \bar{\chi}^{\bar{k}}\right)+R_{j \bar{k} l \bar{p}} \psi^{j} \bar{\psi}^{\bar{k}} \chi^{l} \bar{\chi}^{\bar{p}}
$$

Here,

$$
\nabla \bar{\chi}^{\bar{k}}=\dot{\bar{\chi}}^{\bar{k}}+\dot{\bar{z}}^{\bar{p}} \Gamma_{\bar{p} \bar{j}}^{\bar{k}} \bar{\chi}^{\bar{j}}, \quad \nabla \chi^{j}=\dot{\chi}^{j}+\dot{z}^{l} \Gamma_{l p}^{j} \chi^{p}
$$

and $R_{j \bar{k} l \bar{p}}$ is the Riemann tensor for the Kähler metric defined in (2.22). Its appearance in the Lagrangian is an important new feature of the $\mathcal{N}=4$ case compared to Eq.(3.20).

\footnotetext{
${ }^{11}$ In this notation, the $\mathcal{N}=2$ multiplets corresponding to the superfields $Z^{i}$ and $\Phi^{i}$ can be denoted as $(\mathbf{2}, \mathbf{2}, \mathbf{0})$ and $(\mathbf{0}, \mathbf{2}, \mathbf{2})$.
} 
The total $\mathcal{N}=4$ supersymmetric component Lagrangian can be concisely written as

$$
L=h_{j \bar{k}}\left[\dot{z}^{j} \dot{\bar{z}}^{\bar{k}}+\frac{i}{2}\left(\psi^{j} \nabla \bar{\psi}^{\bar{k}}+\chi^{j} \nabla \bar{\chi}^{\bar{k}}-\nabla \psi^{j} \bar{\psi}^{\bar{k}}-\nabla \chi^{j} \bar{\chi}^{\bar{k}}\right)\right]+R_{j \bar{k} l \bar{p}} \psi^{j} \bar{\psi}^{\bar{k}} \chi^{l} \bar{\chi}^{\bar{p}} .
$$

The $\mathcal{N}=4$ supersymmetry closes on shell, since we have eliminated the auxiliary fields $d^{j}, \bar{d}^{\bar{k}}$.

The Lagrangian (4.11) is well known. It coincides with the Lagrangian obtained by deleting spatial derivatives in the $(1+1)$-dimensional $\mathcal{N}=2 \sigma$-model Lagrangian 41] and discussed, e.g., in [42, 43, 32] (there, fermionic fields $\psi^{j}, \chi^{j}$ were combined into a $\mathrm{SU}(2)$ doublet). We refer the reader to 32 for the expressions for the classical and quantum supercharges, the Hamiltonian, etc.

It is worth also recalling that the Lagrangian (4.11) coincides with the generic SQM sigma-model Lagrangian involving $D$ supermultiplets $(\mathbf{1}, \mathbf{2}, \mathbf{1}$ ) [44, 45, 46],

$$
L=g_{M N}\left(\frac{1}{2} \dot{z}^{M} \dot{z}^{N}+i \bar{\psi}^{M} \nabla \psi^{M}\right)+\frac{1}{2} R_{M N P Q} \bar{\psi}^{M} \psi^{N} \bar{\psi}^{P} \psi^{Q} .
$$

For a generic metric, the latter Lagrangian enjoys only $\mathcal{N}=2$ supersymmetry, but in the Kähler case, a second pair of supercharges can be found. Note also that, when an external gauge field is present, there is no such second pair. A related almost equivalent statement is that no $\mathcal{N}=4$ completion based on the linear chiral $\mathcal{N}=4, d=1$ multiplets $(\mathbf{2}, \mathbf{4}, \mathbf{2})$ is possible for the theory 3.5 with $W \neq 0$. Note that such a completion becomes possible, if extending the $\mathcal{N}=2$ chiral multiplets $(\mathbf{2}, \mathbf{2}, \mathbf{0})$ to nonlinear versions of the $\mathcal{N}=4$ multiplets $(\mathbf{2}, \mathbf{4}, \mathbf{2})$ or $(\mathbf{4}, \mathbf{4}, \mathbf{0})$ [47, 48, 36].

\section{Quantum supercharges and geometry}

Let us assume that $\operatorname{det} \bar{e}=\operatorname{det} e=\sqrt{\operatorname{det} h} 12$ and choose

$$
W=\frac{q}{2(n+1)} \ln \operatorname{det} h \text {. }
$$

Then the general supercharges (3.26) take the form (3.41), (3.42) where we should replace $\omega_{k, \bar{a} b} \rightarrow \Omega_{k, \bar{a} b}$ and

$$
\begin{aligned}
& \frac{\bar{z}^{k}}{1+z \bar{z}} \rightarrow-\frac{1}{n+1} \partial_{k}(\ln \operatorname{det} h) \\
& \frac{z^{k}}{1+z \bar{z}} \rightarrow-\frac{1}{n+1} \partial_{\bar{k}}(\ln \operatorname{det} h) .
\end{aligned}
$$

We see that there are special values $q= \pm(n+1) / 2$ where either $\tilde{\Pi}_{k}$ or $\overline{\tilde{\Pi}}_{k}$ coincide with the usual holomorphic or antiholomorphic derivatives. Consider first the case

\footnotetext{
${ }^{12}$ Such a choice amounts to fixing a gauge with respect to the local frame U(1) transformations of the vielbeins.
} 
$q=(n+1) / 2$. It is not difficult to check that the action of $Q^{\text {cov }}$ on the wave functions

$$
\Psi\left(z^{k}, \bar{z}^{k} ; \psi^{a}\right)=A^{(0)}\left(z^{k}, \bar{z}^{k}\right)+\psi^{a} A_{a}^{(1)}\left(z^{k}, \bar{z}^{k}\right)+\ldots+\psi^{a_{1}} \cdots \psi^{a_{n}} A_{\left[a_{1} \cdots a_{n}\right]}^{(n)}\left(z^{k}, \bar{z}^{k}\right)
$$

is isomorphic to the action of the exterior holomorphic derivative $\partial$ on the set of $n+1$ holomorphic (p,0)-forms (the term $\propto \Omega$ in $Q^{\text {cov }}$ cancels out the term coming from differentiation of the vielbeins in virtue of the structure equation $(2.14)$ ). The Hermitian-conjugate operator $\bar{Q}^{\text {cov }}$ is then isomorphic to $\partial^{\dagger}$. In other words, in this case the supercharges (3.41) realize the standard untwisted (i.e. involving no additional gauge field) Dolbeault complex.

Likewise, in the case $q=-(n+1) / 2$, the action of the operator $\bar{Q}^{\text {cov }}$ on antiholomorphic wave functions $\Psi\left(z^{k}, \bar{z}^{k} ; \bar{\psi}^{a}\right)$ is isomorphic to the action of the operator $\bar{\partial}$ on antiholomorphic (0,p)-forms, the operator $Q^{\text {cov }}$ playing the role of $\bar{\partial}^{\dagger}$. Thus, in this case we are dealing with the anti-holomorphic untwisted Dolbeault complex.

For any other value of $q$, an extra Abelian gauge field is present in the framework of both the holomorphic and antiholomorphic Dolbeault interpretations, i.e.

$$
A_{k}=\frac{i}{4}\left(1-\frac{2 q}{n+1}\right) \partial_{k} \ln \operatorname{det} h
$$

in the holomorphic case and

$$
A_{\bar{k}}=\frac{i}{4}\left(1+\frac{2 q}{n+1}\right) \partial_{\bar{k}} \ln \operatorname{det} h
$$

in the antiholomorphic case. We face what is called twisted Dolbeault complex.

Until now we dealt with the generic (non-Kähler) $\mathcal{N}=2$ SQM model, the only restriction was the relation (5.1). If the manifold is Kähler, the supercharges admit another even more interesting geometric interpretation: when $q=0$, the $\operatorname{sum} Q^{\text {cov }}+$ $\bar{Q}^{\text {cov }}$ can be interpreted as the untwisted Dirac operator. When $q \neq 0$, an extra Abelian gauge field is present.

Indeed, the standard untwisted Dirac operator in the real notations is [cf. (3.32)]

$$
\not D=\gamma^{A} e_{A}^{M}\left(\partial_{M}+\frac{1}{4} \Omega_{M, B C} \gamma^{B} \gamma^{C}\right) \equiv \gamma^{M} \mathcal{D}_{M}
$$

When splitting $M \equiv(k, \bar{k}), \quad A \equiv(a, \bar{a})$ and introducing $\sqrt{2} \psi^{a} \equiv \gamma^{a}, \sqrt{2} \bar{\psi}^{\bar{a}} \equiv \gamma^{\bar{a}}$, one can be convinced that, for Kähler manifolds, one can represent

$$
\not{D}=\mathcal{D}^{\mathrm{Hol}}-\left(\mathcal{D}^{\mathrm{Hol}}\right)^{\dagger}
$$

where

$$
\not{P}^{\mathrm{Hol}}=\sqrt{2} \psi^{b} e_{b}^{k}\left[\partial_{k}+\frac{1}{2} \omega_{k, \bar{a} d}\left(\bar{\psi}^{\bar{a}} \psi^{d}-\psi^{d} \bar{\psi}^{\bar{a}}\right)\right]
$$

and

$$
\left(\not{P}^{\mathrm{Hol}}\right)^{\dagger}=-\sqrt{2} \bar{\psi}^{\bar{b}} e_{\bar{b}}^{\bar{k}}\left[\partial_{\bar{k}}+\frac{1}{2} \bar{\omega}_{\bar{k}, a \bar{d}}\left(\psi^{a} \bar{\psi}^{\bar{d}}-\bar{\psi}^{\bar{d}} \psi^{a}\right)\right]
$$


These operators coincide, up to the factor $i$, with the supercharges (3.26), (3.27) in which one chooses $\Omega=\omega$ and $W=0$ :

$$
\not{D}^{\mathrm{Hol}}=i Q^{\text {cov }}, \quad\left(\not{D}^{\mathrm{Hol}}\right)^{\dagger}=-i \bar{Q}^{\mathrm{cov}}, \quad\left(C_{k i \bar{l}}=q=0\right)
$$

For $q \neq 0$, an additional Abelian gauge field is present, and we are facing the twisted Dirac operator in this case. Note that the definition of "twisting" or "untwisting" is different in the interpretations in terms of Dolbeault and Dirac complexes. E.g., the choice $q=0$ corresponds to an untwisted Dirac complex, but to the twisted Dolbeault complex (as is seen from (5.4), (5.5) ).

The operator $\not \mathcal{D}$ is anti-Hermitian. Consider now the real part of $\mathcal{D}^{\mathrm{Hol}}$,

$$
S=\not{D}^{\mathrm{Hol}}+\left(\mathcal{D}^{\mathrm{Hol}}\right)^{\dagger}
$$

One can be convinced that, instead of $\sqrt{2} \psi^{M} \mathcal{D}_{M} \equiv \gamma^{M} \mathcal{D}_{M}$ for the imaginary (antiHermitian) part of $\not^{\text {Hol }}$, for the real (Hermitian) part we obtain the expression

$$
S=-i \gamma^{N} I_{N}^{M} \mathcal{D}_{M}
$$

This is immediately seen when writing the components of the complex structure tensor (1.5) in the complex basis: $I_{n}^{m}=i \delta_{n}^{m} ; I_{\bar{n}}^{\bar{m}}=-i \delta_{\bar{n}}^{\bar{m}}$. The pair of supercharges (1.4) is thus reproduced.

A by-product of this analysis is a physical proof of the purely mathematical fact: for Kähler manifolds, the twisted Dirac complex is equivalent to the twisted Dolbeault complex, bearing in mind that the twisting (the adding of Abelian gauge fields) in the Dirac complex and in the Dolbeault complex is different. This fact is known to mathematicians, see e.g. the Propositions 1.4.23 and 1.4.25 in the book [49].

If the manifold is not Kähler, the decomposition (5.7) - (5.9) is no longer valid. However, as was noted above, the sum $\bar{Q}+Q$ of the quantum supercharges (3.26) can be represented as the Dirac operator with some special torsions (3.35). The imaginary part $S \propto \bar{Q}-Q$ may be obtained from (3.35) by commuting (3.35) with the fermion charge operator $F=\frac{i}{2} I_{M N} \psi^{M} \psi^{N}=\frac{i}{4} I_{M N} \gamma^{M} \gamma^{N}$. The result is [50]

$$
S=\sqrt{2} \psi^{N} I_{N}^{M}\left[\Pi_{M}-\frac{i}{2} \Omega_{M, B C} \psi^{B} \psi^{C}-\frac{i}{4} C_{M K L} \psi^{K} \psi^{L}\right]
$$

\section{Index}

The Euclidean path integral representation for the index (1.3) of our system is

$$
\begin{aligned}
I= & \int \prod_{j \tau} \frac{d \pi_{j}(\tau) d \bar{\pi}_{\bar{j}}(\tau) d z^{j}(\tau) d \bar{z}^{\bar{j}}(\tau)}{(2 \pi)^{2}} \prod_{a \tau} d \psi^{a}(\tau) d \bar{\psi}^{\bar{a}}(\tau) \\
& \times \exp \left\{\int_{0}^{\beta} d \tau\left[i \pi_{j} \dot{z}^{j}+i \bar{\pi}_{j}^{-} \dot{\bar{z}}^{\bar{j}}+\dot{\bar{\psi}}^{\bar{a}} \psi^{a}-H\left(\pi_{j}, \bar{\pi}_{\bar{j}}, z^{j}, \bar{z}^{\bar{j}} ; \bar{\psi}^{\bar{a}}, \psi^{a}\right)\right]\right\},
\end{aligned}
$$


where both bosonic and fermionic variables satisfy the periodic boundary conditions, $z^{j}(\beta)=z^{j}(0)$, etc. Expand all the variables in the Fourier series,

$$
z^{j}(\tau)=z^{j(0)}+\sum_{m \neq 0} z^{j(m)} e^{2 \pi i m \tau / \beta} \quad \bar{z}^{\bar{j}}(\tau)=z^{\bar{j}(0)}+\sum_{m \neq 0} \bar{z}^{\bar{j}(m)} e^{-2 \pi i m \tau / \beta},
$$

and similarly for $\pi_{j}(\tau), \bar{\pi}_{\bar{j}}(\tau)$ and $\psi^{a}(\tau), \bar{\psi}^{\bar{a}}(\tau)$. If $\beta$ is small, we seemingly (see below) can neglect the nonzero modes in the expansion, neglect thereby the terms with time derivatives in (6.1), and rewrite (6.1) as an ordinary integral [51, 52]:

$$
\begin{aligned}
I= & \int \prod_{j} \frac{d \pi_{j}^{(0)} d \bar{\pi}_{\bar{j}}^{(0)} d z^{j(0)} d \bar{z}^{j(0)}}{(2 \pi)^{2}} \prod_{a} d \psi^{a(0)} d \bar{\psi}^{\bar{a}(0)} \\
& \times \exp \left\{-\beta H\left(\pi_{j}^{(0)}, \bar{\pi}_{\bar{j}}^{(0)}, z^{j(0)}, \bar{z}^{j(0)} ; \psi^{a(0)}, \bar{\psi}^{\bar{a}(0)}\right)\right\} .
\end{aligned}
$$

The functional integral is reduced to the ordinary one in the semiclassical limit $\beta \rightarrow 0$. However, the index (1.3) does not depend on $\beta$, and the estimate (6.3) should be true for any $\beta$.

Substituting here the Hamiltonian (3.17) with the choice (5.1) (remember that, for Kähler manifolds, the last term in (3.17) vanishes), we can easily integrate over $\prod_{j} d \pi_{j} d \bar{\pi}_{\bar{j}}$ and over $\prod_{a} d \psi^{a} d \bar{\psi}^{\bar{a}}$ to obtain

$$
I=\left(\frac{1}{2 \pi}\right)^{n} \int \prod_{j} d z^{j} d \bar{z}^{\bar{j}} \operatorname{det}\left\|h_{j \bar{k}}\right\| \operatorname{det}\left\|i \mathcal{F}_{a \bar{b}}\right\|,
$$

where $i \mathcal{F}_{a \bar{b}}=-2 e_{a}^{j} e_{\bar{b}}^{\bar{k}} \partial_{j} \partial_{\bar{k}} W$ is related to the 2-form describing the magnetic field strength. In the simplest $\mathbb{C P}^{n}$ case under the choice (5.1), we have $i \mathcal{F}_{a \bar{b}}=q \delta_{a \bar{b}}$ leading to

$$
I=\left(\frac{q}{2 \pi}\right)^{n} \int \prod_{j} d z^{j} d \bar{z}^{j}(\operatorname{det} h) .
$$

The calculation with the Fubini-Study metric (3.38), i.e. with $\operatorname{det} h=\frac{1}{(1+z \bar{z})^{n+1}}$, gives

$$
I_{\mathbb{C P}^{n}} \stackrel{?}{=} \frac{q^{n}}{n !} .
$$

This result looks suspicious. Indeed, to make it integer (the index should be integer for the Dirac operator to make sense: only in this case the manifold admits spin structure), $q$ should depend on $n$ in an odd way.

Actually, the estimate (6.6) is wrong. The correct estimate reads [2, 3, 4, 5, 14, 15]

$$
I=\int e^{\mathcal{F} / 2 \pi} \operatorname{det}^{-1 / 2}\left[\frac{\sin \frac{\mathcal{R}}{4 \pi}}{\frac{\mathcal{R}}{4 \pi}}\right],
$$

where $\mathcal{F}$ is the field strength 2 -form and $\mathcal{R}$ is the matrix 2-form associated with the Riemann curvature,

$$
\mathcal{F}=\frac{1}{2} F_{M N} d x^{M} \wedge d x^{N}, \quad \mathcal{R}^{A B}=\frac{1}{2} R_{M N}^{A B} d x^{M} \wedge d x^{N}
$$


$A, B$ being the tangent space indices.

The precise meaning of the representation (6.7) is that the volume integral in its r.h.s. projects out only the forms of the maximal rank $D$ from the Taylor expansion of the integrand. Thus, for 4-dimensional manifolds, the index is represented as the sum of two terms,

$$
I_{d=4}=\frac{1}{8 \pi^{2}} \int \mathcal{F} \wedge \mathcal{F}+\frac{1}{192 \pi^{2}} \int \operatorname{Tr}\{\mathcal{R} \wedge \mathcal{R}\} .
$$

The topological invariants in the r.h.s. are known as the second Chern class $c_{2}$ and the Hirzebruch signature $\tau$ (the latter enters with the coefficient -1/8). For higher dimensions, the index is a sum of many different invariants.

It is convenient to represent the determinant factor in (6.7) as

$$
\operatorname{det}^{-1 / 2}[\cdots]=\prod_{\alpha=1}^{n} \frac{\lambda_{\alpha} /(4 \pi)}{\sinh \left(\lambda_{\alpha} /(4 \pi)\right.},
$$

where $\lambda_{\alpha}$ are the eigenvalues of the antisymmetric matrix $\mathcal{R}^{A B}$. This can be derived by diagonalizing,

$$
\mathcal{R} \longrightarrow\left(i \sigma_{2} \lambda_{1}, \ldots, i \sigma_{2} \lambda_{n}\right),
$$

and noting that, for any even function $f(\mathcal{R})$,

$$
\operatorname{det}^{-1 / 2} f(\mathcal{R})=\prod_{\alpha=1}^{n} \frac{1}{f\left(i \lambda_{\alpha}\right)} .
$$

The estimate (6.6) for $\mathbb{C P}^{n}$ would be reproduced, if ignoring this curvaturedependent determinant factor in (6.7). When including this factor, we obtain instead

$$
I_{\mathbb{C P}^{n}}=\left(\begin{array}{c}
q+(n-1) / 2 \\
n
\end{array}\right),
$$

where $q$ must be integer for odd $n$ and half-integer for even $n$. The index is given by Eq.(6.11) when $q \geq(n+1) / 2$. For negative $q \leq-(n+1) / 2$, it is given by

$$
I_{\mathbb{C P}^{n}}(q<0)=(-1)^{n}\left(\begin{array}{c}
|q|+(n-1) / 2 \\
n
\end{array}\right) .
$$

The index vanishes for $|q|<(n+1) / 213$.

The result (6.11) for the index in $\mathbb{C P}^{n}$ can also be derived directly, simply by counting the number of independent ground states [56, 21, i.e. the number of the normalized (with the measure (3.24), in which $\operatorname{det} h=1 /(1+z \bar{z})^{n+1}$ ) solutions to the equations

$$
Q^{c o v} \Psi_{0}=\bar{Q}^{c o v} \Psi_{0}=0,
$$

\footnotetext{
${ }^{13}$ Note in passing that the index (6.11) is closely related to the Witten index in $3 \mathrm{~d}$ supersymmetric Yang-Mills-Chern-Simons theory [53. See [54, 55] for detailed discussion.
} 
with $Q^{\text {cov }}$ and $\bar{Q}^{\text {cov }}$ defined in (3.41), (3.42). Choosing, e.g., the holomorphic representation (5.3) for the wave functions, we find that, in the sector of zero fermionic charge, the equation $\bar{Q}^{\operatorname{cov}} \Psi_{0}=0$ is satisfied identically, while the equation $Q^{\operatorname{cov}} \Psi_{0}=0$ implies

$$
\partial_{k} \Psi_{0}=-\frac{s \bar{z}^{\bar{k}}}{1+z \bar{z}} \Psi_{0}
$$

with

$$
2 s=q-\frac{n+1}{2} .
$$

We see that the normalized solutions exist only at $s \geq 0$. They have the form

$$
\Psi_{0}=\Psi(z, \bar{z})=(1+z \bar{z})^{-s} \Phi(\bar{z})
$$

where $\Phi(\bar{z})$ is a polynomial of $\bar{z}^{\bar{j}}$ of the rank not higher than $2 s$. Then the number of independent ground states is given by the binomial coefficient

$$
\frac{(n+2 s) !}{n !(2 s) !}=\frac{\left(q+\frac{n-1}{2}\right) !}{n !\left(q-\frac{n+1}{2}\right) !},
$$

which exactly coincides with Eq. (6.11). For negative $q$, the vacuum states are present in the sector of fermion charge $F=n$, hence the factor $(-1)^{n}$ in Eq. (6.12).

What was wrong then in the calculation having led to (6.6)? The answer is that the recipe [51, 52] that allowed us to replace the functional integral (6.1) by the ordinary integral (6.3) and that works well for many SQM and supersymmetric field theory systems fails in this case. To obtain the correct estimate for the index, one should take into account the nonzero Fourier modes in the expansion (6.2) and integrate them over in the Gaussian (see below) approximation. This integral gives exactly the determinant factor in (6.7).

To perform the actual calculation 14 , we assume $\beta$ to be small, impose periodic boundary conditions, subdivide the interval $(0, \beta)$ into a large number $N$ of integration points and integrate first over $\prod_{j \tau} \frac{d \pi_{j}(\tau) d \bar{\pi}_{\bar{j}}(\tau)}{2 \pi}$ to obtain

$$
\begin{aligned}
I= & \int \prod_{\tau} \operatorname{det} h\left(\bar{z}^{\bar{j}}(\tau), z^{j}(\tau)\right) \prod_{j} \frac{d \bar{z}^{j}(\tau) d z^{j}(\tau)}{2 \pi(\beta / N)} \prod_{a} d \psi^{a}(\tau) d \bar{\psi}^{\bar{a}}(\tau) \\
& \times \exp \left\{-\int_{0}^{\beta} L_{E}(\tau) d \tau\right\},
\end{aligned}
$$

with

$$
L_{E}=h_{j \bar{k}} \dot{z}^{j} \dot{\bar{z}}^{\bar{k}}+\frac{1}{2}\left(\psi^{a} \dot{\bar{\psi}}^{\bar{a}}-\dot{\psi}^{a} \bar{\psi}^{a}\right)+\left(\dot{\bar{z}}^{\bar{k}} \omega_{\bar{k}, a \bar{b}}-\dot{z}^{k} \omega_{k, \bar{b} a}\right) \psi^{a} \bar{\psi}^{\bar{b}}
$$

The product $\prod_{\tau}$ in (6.18) runs over $N$ discrete points $\tau_{r}=\beta r / N, \quad r=0, \ldots, N-1$. For simplicity, we suppressed the gauge part that was already successfully handled

\footnotetext{
${ }^{14}$ It is rather similar in spirit to the calculation of the functional integral for SQM describing the

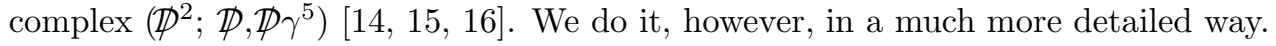


earlier by the Cecotti-Girardello method. It is the determinant factor depending only on the Riemannian manifold geometry that is of interest for us now.

Substitute now the expansion (6.2) into (6.19). If the number of points $N$ is large but finite, we have also to keep the number of Fourier modes finite, so that the sum in (6.2) runs over $m=-M, \ldots, 0, \ldots, M$, where $N=2 M+1$. To calculate the functional integral in the Gaussian approximation 15 (we will justify the validity of this approximation later), we keep only quadratic (in $\bar{z}_{m}^{\bar{j}}, z_{m}^{j}, \bar{\psi}_{m}^{\bar{a}}$ and $\psi_{m}^{a}$ ) terms and do the $\tau$-integral. The quadratic part of the Lagrangian gives

$$
\begin{aligned}
\int_{0}^{\beta} L_{E}^{(2)} d \tau= & -i \beta \sum_{m}^{\prime} \Omega_{m} \psi_{m}^{a} \bar{\psi}_{m}^{\bar{a}}-i \beta \sum_{m}^{\prime} \Omega_{m}\left[\psi^{a(0)} \omega_{k, \bar{b} a}^{(0)} \bar{\psi}_{m}^{\bar{b}} z_{m}^{k}-\bar{\psi}^{\bar{b}(0)} \psi_{m}^{a} \bar{z}_{m}^{\bar{k}} \omega_{\bar{k}, a \bar{b}}^{(0)}\right] \\
& +\beta \sum_{m}^{\prime} z_{m}^{j} \bar{z}_{m}^{\bar{k}}\left[\Omega_{m}^{2} h_{j \bar{k}}^{(0)}-i \Omega_{m} \psi^{a(0)} \bar{\psi}^{\bar{b}(0)}\left(\partial_{j} \omega_{\bar{k}, a \bar{b}}^{(0)}+\partial_{\bar{k}} \omega_{j, \bar{b} a}^{(0)}\right)\right] \\
& -i \beta \sum_{m}^{\prime} \Omega_{m}\left[\psi^{a(0)} \omega_{\bar{k}, a \bar{b}}^{(0)} \bar{z}_{m}^{\bar{k}} \bar{\psi}_{-m}^{\bar{b}}-\bar{\psi}^{\bar{a}(0)} z_{m}^{k} \psi_{-m}^{b} \omega_{k, \bar{a} b}^{(0)}\right] \\
& -i \beta \sum_{m}^{\prime} \Omega_{m} \psi^{a(0)} \bar{\psi}^{\bar{b}(0)}\left[\partial_{k} \omega_{j, \bar{b} a}^{(0)} z_{m}^{j} z_{-m}^{k}+\partial_{\bar{k}} \omega_{\bar{j}, a \bar{b}}^{(0)} \bar{z}_{m}^{\bar{j}} \bar{z}_{-m}^{\bar{k}}\right]
\end{aligned}
$$

with $\Omega_{m}=2 \pi m / \beta$. The sum $\sum_{m}^{\prime}$ runs over all nonzero modes. When writing this, we assumed $m \ll M$. If $m \sim M$, one is not allowed to approximate the finite differences in the Euclidean action entering the finite-number-of-point approximation (6.18) of the path integral by time derivatives. An accurate analysis displays that the only change one should implement for large $m$ is to substitute

$$
\Omega_{m} \longrightarrow \tilde{\Omega}_{m}=-i \frac{N}{\beta}\left(1-e^{-2 \pi i m / N}\right)
$$

in Eq.(6.20). However, as we will see later, this replacement affects only the overall coefficient in the functional integral that is fixed separately, while the nontrivial dependence of the integrand on the metric is determined by the contribution of only first few Fourier modes.

Thus, we keep for the moment $\Omega_{m}=2 \pi m / \beta$ and diagonalize the sum in (6.20) by the substitution

$$
\begin{aligned}
\psi_{m}^{b} & \Rightarrow \psi_{m}^{b}+\psi^{a(0)}\left[\bar{z}_{-m}^{\bar{k}} \omega_{\bar{k}, a \bar{b}}^{(0)}-z_{m}^{k} \omega_{k, \bar{b} a}^{(0)}\right], \\
\bar{\psi}_{m}^{\bar{b}} & \Rightarrow \bar{\psi}_{m}^{\bar{b}}+\bar{\psi}^{\bar{a}(0)}\left[z_{-m}^{k} \omega_{k, \bar{a} b}^{(0)}-\bar{z}_{m}^{\bar{k}} \omega_{\bar{k}, b \bar{a}}^{(0)}\right] .
\end{aligned}
$$

It brings (6.20) to the simple form

$$
\int_{0}^{\beta} L_{E}^{(2)} d \tau=\sum_{m}^{\prime} A_{m a \bar{b}} \psi_{m}^{a} \bar{\psi}_{m}^{\bar{b}}+\sum_{m}^{\prime} D_{m j \bar{k}} z_{m}^{j} \bar{z}_{m}^{\bar{k}},
$$

\footnotetext{
${ }^{15}$ Incidentally, the result (6.18) can also be reproduced by trading the variables $\pi_{j}\left(\tau_{r}\right)$ and $\bar{\pi}_{j}\left(\tau_{r}\right)$ for their Fourier modes and performing then the Gaussian integral over $\prod_{m}^{\prime} d \pi_{j}^{(m)} d \bar{\pi}_{\bar{j}}^{(m)}$. In this case, the factor $N^{N}$ seen in (6.18) appears as the Jacobian of the variable change (6.2).
} 
where

$$
A_{m a \bar{b}}=-i \beta \Omega_{m} \delta_{a \bar{b}}, \quad D_{m j \bar{k}}=\beta\left[\Omega_{m}^{2} h_{j \bar{k}}^{(0)}-i \Omega_{m} R_{j \bar{k}, a \bar{b}} \psi^{a(0)} \bar{\psi}^{\bar{b}(0)}\right]
$$

and $R_{j \bar{k}, a \bar{b}}$ is the Riemann tensor defined in (2.22). In the process of passing from (6.20) to (6.23) we used the property $\Omega_{-m}=-\Omega_{m}$ and also the identity

$$
\partial_{[l} \omega_{k], \bar{b} a}-\omega_{[l, \bar{d} a} \omega_{k], \bar{b} d}=0 \quad \text { (and c.c.) }
$$

(the 1.h.s. of Eq.(6.25) is none other than the component $R_{l k \bar{b} a}$ of the Riemann tensor that vanishes for Kähler manifolds).

Note that the matrix of the partial derivatives corresponding to the substitution (6.22) is triangle and so has a unit superdeterminant. The super-Jacobian for the variable change (6.2) is also equal to unity, because the bosonic and fermion determinants cancel each other. The functional integral over non-zero modes is then given by a product of a large (in the continuous limit, infinite) number of finite-dimensional determinants, which can be symbolically written as

$$
\begin{aligned}
\text { grav. factor } & =\mu \prod_{m}^{\prime} \prod_{j} \frac{d z_{m}^{j} d \bar{z}_{m}^{\bar{j}}}{2 \pi} \prod_{a} d \psi_{m}^{a} d \bar{\psi}_{m}^{\bar{a}} \exp \left\{-A_{m} \psi_{m} \bar{\psi}_{m}-D_{m} z_{m} \bar{z}_{m}\right\} \\
& =\mu \prod_{m}^{\prime} \operatorname{det}\left\|A_{m}\right\| \cdot \operatorname{det}^{-1}\left\|D_{m}\right\|
\end{aligned}
$$

where

$$
\mu=\left(\operatorname{det}\left\|h_{i \bar{k}}^{(0)}\right\|\right)^{N} \prod_{m=1}^{M} \Omega_{m}^{2 n}
$$

is the appropriate measure. The factor $\left(\operatorname{det}\left\|h_{i \bar{k}}^{(0)}\right\|\right)^{N}$ in (6.27) comes from the factor $\prod_{r=0}^{N-1} \operatorname{det}\left\|h_{i \bar{k}}\left(\tau_{r}\right)\right\|$ in (6.18) , where the dependence of $z^{j}(\tau), \bar{z}^{\bar{j}}(\tau)$ on higher Fourier harmonics has been suppressed. This suppression can be justified by noticing that the characteristic values of $z_{m}$ in the integral $\prod_{m j} d z_{m}^{j} d \bar{z}_{m}^{\bar{j}} \exp \left\{-\int_{0}^{\beta} L_{E}^{(2)} d \tau\right\}$ are $z_{m} \sim$ $1 / \Omega_{m} \sqrt{\beta} \sim \sqrt{\beta}$, which is small at small $\beta$. The dimensional factor $\beta^{-2 M n}$ in (6.27) comes from the factor $\beta^{-n N}=\beta^{-2 n M} \times \beta^{-n}$ in (6.18) (the factor $\beta^{-n}$ having been borrowed to be displayed in the constant mode integral (6.3) after performing the integration over momenta). To derive from (6.18) the correct numerical factor in the measure, notice that the coefficient $N^{N}$ present in (6.18) can be represented as

$$
N^{N}=\prod_{m=1}^{M}\left(\beta \tilde{\Omega}_{m}\right)\left(\beta \tilde{\Omega}_{-m}\right)
$$

which follows in turn from the known identity 16

$$
\prod_{r=1}^{N-1}\left(1-w^{r}\right)=N, \quad \text { if } w=e^{2 \pi i / N} .
$$

\footnotetext{
${ }^{16}$ Consider $P(x)=x^{N}-1=\prod_{r=0}^{N-1}\left(x-w^{r}\right)$ and calculate $P^{\prime}(1)$.
} 
Then, bearing in mind that only first few values $m$ are relevant (see below), we can replace $\tilde{\Omega}_{m} \rightarrow \Omega_{m}$, which yields (6.27). It is much easier, of course, to fix the factor in (6.27) from the condition that the r.h.s. of Eq.(6.26) is equal to 1 in the flat case $h_{j \bar{k}}=\delta_{j \bar{k}}$.

The calculation gives

$$
\begin{aligned}
\text { grav. factor } & =\left(\operatorname{det}\left\|h_{i \bar{k}}^{(0)}\right\|\right)^{N} \prod_{m}^{\prime} \frac{\Omega_{m}^{2 n}}{\operatorname{det}\left\|h_{i \bar{k}}^{(0)}\right\| \operatorname{det}\left\|\Omega_{m}^{2} \delta_{j}^{q}-i \Omega_{m} R_{j}^{q}\right\|} \\
& =\operatorname{det}\left\|h_{i \bar{k}}^{(0)}\right\| \prod_{m=1}^{\infty} \frac{\Omega_{m}^{2 n}}{\operatorname{det}\left\|\Omega_{m}^{2} \delta_{j}^{q}+R_{j}^{s} R_{s}^{q}\right\|}
\end{aligned}
$$

where

$$
R_{j}^{q}=h^{\bar{k} q} R_{j \bar{k} l} \psi^{l(0)} \bar{\psi}^{\bar{p}(0)}
$$

and we took into account the relation $N=2 M+1$ and sent $M \rightarrow \infty$ afterwards. We see that only one power of the determinant $\operatorname{det} h^{(0)}$ is left.

The infinite $m$-product in (6.30) can be done by writing the determinant as the product of the eigenvalues and using the identity

$$
\prod_{m=1}^{\infty} \frac{(2 \pi m)^{2}}{(2 \pi m)^{2}+a^{2}}=\frac{a}{2 \sinh (a / 2)} .
$$

For $a \sim 1$, only few first values of $m$ are essential in this product, and it justifies as promised the assumption $m \ll M$ under which Eq.(6.20) was derived.

We finally obtain

$$
I^{\text {pure gravity }}=\frac{1}{(2 \pi \beta)^{n}} \int \prod_{j=1}^{n} d \bar{z}^{\bar{j}} d z^{j} d \psi^{j} d \bar{\psi}^{\bar{j}} \operatorname{det} \frac{\beta R / 2}{\sinh (\beta R / 2)},
$$

where we suppressed the superscripts ${ }^{(0)}$ and passed back to the integration over the fermionic zero modes with the world indices $\psi^{j}, \bar{\psi}^{j}$ (this absorbs the remaining factor det $h$ in (6.30) $)$. Multiplying the integrand by $\exp \left\{-i \beta \mathcal{F}_{j \bar{k}} \psi^{j} \bar{\psi}^{\bar{k}}\right\}$ and doing the fermion integral, we arrive at (6.10) and hence to (6.7) 17. It is clear now why, in this particular case, we had to insert the 1-loop gravitational factor in the tree-level integral (6.3) for the index. Formally, the factor (6.30) tends to 1 for small $\beta$ and, naively, the corrections involving $\beta$ and its higher powers can be neglected. We see,

\footnotetext{
17 To establish the exact correspondence, one has to keep in mind that the skew-symmetric matrix
} $\mathcal{R}$ defined in (6.8) is represented in the Kähler case as

$$
\mathcal{R}^{A B}=\left(\begin{array}{cc}
0 & -R^{a \bar{b}} \\
R^{b \bar{a}} & 0
\end{array}\right)
$$

where

$$
R^{a \bar{b}}=e_{i}^{a} e_{\bar{k}}^{\bar{b}} R_{j \bar{t}}^{i \bar{k}} d z^{j} \wedge d \bar{z}^{\bar{t}}
$$


however, that each factor $\beta$ in the expansion is multiplied by a bi-fermion structure $\sim \psi \bar{\psi}$, as is also the case for the expansion of the integrand in (6.3). For the fermion integral not to vanish, we have to pick up the terms $\sim \beta^{n}(\psi \bar{\psi})^{n}$ in the expansion of both the factor $\exp \{-i \beta \mathcal{F} \psi \bar{\psi}\}$ inside the tree-level integral and of the 1-loop factor (6.30) - they come on equal footing.

On the other hand, the possible semiclassical corrections involving more powers of $\beta$ than those coming from $\psi \bar{\psi}$ are not relevant (cf. a remark in the paragraph after (6.27)). This justifies neglecting two-loop and higher-loop effects in the functional integral (6.1).

\section{Final comments and summary}

In the previous Section, we have constructed the proof of the Atiyah-Singer theorem for the standard Dirac operator based on the analysis of our SQM model for the Kähler manifolds and for Abelian gauge fields. The same method can be and was used, however, to prove it for any even-dimensional manifold. To this end, one should consider the system defined by the Lagrangian (3.31) accompanied by the external gauge field Lagrangian (3.10).

As was discussed above, in the generic case, the $\mathcal{N}=2$ supersymmetry algebra is realized not by the supercharges (1.4), but by the supercharges $\not{D}$ and $\not D \gamma^{D+1}$. The Witten index (1.3) of this model still coincides with the Atiyah-Singer index of $\not{D}$. One can be easily convinced in it by introducing the holomorphic variables $\chi^{1}=\psi^{1-i 2} / \sqrt{2}, \chi^{2}=\psi^{3-i 4} / \sqrt{2}$, etc, and noting that

$$
\gamma^{D+1} \equiv(2 i)^{D / 2} \prod_{A=1}^{D} \psi^{A}=\prod_{a=1}^{D / 2}\left(\bar{\chi}^{a} \chi^{a}-\chi^{a} \bar{\chi}^{a}\right) \equiv(-1)^{F} .
$$

Then we have to expand the Euclidean version of the Lagrangians (3.31) and (3.10) into the modes and to perform basically the same calculation as described above. It gives the same answer (6.7). Exploring somewhat more complicated SQM systems, this method can be generalized to non-Abelian gauge fields too.

For the index of the Dolbeault complex in a generic complex case (called sometimes arithmetic genus by mathematicians), especially, in the case where the torsion form is not closed, the life is much more difficult. 18 The Lagrangian (3.9) involves in this case also the large (with respect to the $\beta$ counting) 4 -fermion term such that one cannot neglect the higher-loop contributions anymore. Starting from the complex dimension 2, two-loop contributions do not vanish, starting from the complex dimension 4, one should also add 3-loop contributions, etc. As a result, a direct evaluation of the functional integral is not possible anymore. Still, one can obtain the integral representation for the index (the so called Hirzebruch-Riemann-Roch theorem) by

\footnotetext{
${ }^{18}$ When the torsion is closed, the calculation is still possible. Such calculation is, in fact, the subject of Ref. 27] (even though this paper is purely mathematical and does not refer to functional integrals, etc) and Ref. 28 .
} 
deforming the system considered in this paper in such a way that the torsions vanish while supersymmetry survives the deformation (see a recent paper [25] for details).

Coming back to the calculation of Sector 6, we want to mention that there is another way to evaluate the curvature-dependent corrections to the naive leading order semiclassical result (6.6). One can proceed in the framework of the Hamiltonian formalism and notice that the index is given by the phase space integral of the Weyl symbol of the operator $e^{-\beta H}$. The point is that, generically, $\left[e^{-\beta H}\right]_{W}$ differs from $e^{-\beta H_{W}}$ and there appear corrections involving higher powers of $\beta$. The simplest correction of this type for a generic SQM system with the phase space variables $\left(p_{j}, q_{j} ; \bar{\psi}_{a}, \psi_{a}\right)$ is expressed as [57]

$$
\left[e^{-\beta H}\right]_{W}=e^{-\beta H_{W}}\left(1+\delta+O\left(\beta^{4}\right)\right)
$$

with

$$
\begin{aligned}
\delta\left(p_{j}, q_{j} ; \psi_{a}, \bar{\psi}_{a}\right)= & \frac{\beta^{2}}{48}\left[\frac{\partial^{2}}{\partial \Psi_{a} \partial \bar{\psi}_{a}}-\frac{\partial^{2}}{\partial \psi_{a} \partial \bar{\Psi}_{a}}+i\left(\frac{\partial^{2}}{\partial q_{j} \partial P_{j}}-\frac{\partial^{2}}{\partial Q_{j} \partial p_{j}}\right)\right]^{2} \\
& \times\left. H\left(p_{j}, q_{j} ; \bar{\psi}_{a}, \psi_{a}\right) H\left(P_{j}, Q_{j} ; \bar{\Psi}_{a}, \Psi_{a}\right)\right|_{P=p, Q=q ; \bar{\Psi}=\bar{\psi}, \Psi=\psi}
\end{aligned}
$$

In most cases, this correction is suppressed at small $\beta$ and so is irrelevant. However, in our case, it gives a relevant $\beta$-independent contribution,

$$
\Delta_{\delta} I=-\frac{1}{96 \pi^{2}} \int \prod_{j} d z^{j} d \bar{z}^{\bar{j}} h^{\bar{l} k} h^{\bar{t} p} \epsilon^{m s} \epsilon^{\bar{n} \bar{q}} R_{k \bar{t} m \bar{n}} R_{p \bar{l} s \bar{q}}=-\frac{\tau}{8},
$$

where $\tau$ is the Hirzebruch signature. This coincides with the second term in (6.9).

We see that the Lagrangian method is much more convenient than the Hamiltonian one: the one-loop correction manifestly seen within the Lagrangian method corresponds to a complicated series in $\beta$ on the Hamiltonian side. To find a relevant $\propto \beta^{4}$ term in the expansion (7.2) is already a pretty difficult task.

Finally, it is worth mentioning that there are also other cases when the index cannot be expressed as the simple phase space integral (6.3). First of all, this concerns the systems with the continuous spectrum, like superconformal quantum mechanics [58] or super-Yang-Mills quantum mechanics, where the integrals like (6.3) give meaningless fractional values [59]-63]. In these cases, due to the absence of the gap, such integrals cannot be "focused" on zero energy normalized states, but are "contaminated" by the states from continuum.

The systems with continuous spectrum are widely known and discussed in the literature. There is, however, another interesting class of systems, the SQM systems related to Abelian [64] and non-Abelian [65] chiral supersymmetric $4 D$ gauge theories. In the latter case, the spectrum seems to be discrete, the index is well-defined, and still the integral (6.3) gives a fractional value. It would be rather interesting to see whether this "anomaly" can be cured by taking into account the 1-loop determinant in the spirit of (6.7).

There is also a problem in the index calculation for "symplectic" supersymmetric $\mathcal{N}=4 \sigma$-models with bosonic part describing the motion over a $3 D$ conformally flat 
manifold [66, 67, 68. For example, for $S^{3}$, the index is equal to 2, while the integral (6.3) gives a meaningless irrational number. One of us has shown in [57] that the corrections to (6.3) are present in this case and that they are of the same order as the tree-level contribution. It would be interesting to try to sum up all such corrections by the Lagrangian functional integral method.

As follows from the text of our paper, it involves both the review of the known facts concerning the interrelations between the complex geometry and $\mathcal{N}=2,1$ supersymmetric quantum mechanics and a considerable amount of the new results in this area. For convenience of the reader, we finish with the short summary of these new findings.

1. We constructed, for the first time, quantum supercharges and Hamiltonian for the $\mathcal{N}=2$ SQM model (3.5) in the case of general complex $n$-dimensional manifold and established the one-to-one correspondence of this system with twisted and untwisted Doulbeault complexes.

2. For the Kähler manifolds, we also found a correspondence with twisted and untwisted Dirac complexes and confirmed the equivalence of the twisted Dirac and Dolbeault complexes for this case.

3. We presented a new detailed calculation of the index of the Dirac operator for the Kähler manifolds within the considered $\mathcal{N}=2 \mathrm{SQM}$ model and thereby gave one more "physical" proof of the Atiyah-Singer theorem for this operator.

4. We presented $\mathcal{N}=2$ superfield formulation of the Kähler $\mathcal{N}=4$ SQM model based on the off-shell $\mathcal{N}=4$ multiplet $(\mathbf{2}, \mathbf{4}, \mathbf{2})=(\mathbf{2}, \mathbf{2}, \mathbf{0}) \oplus(\mathbf{0}, \mathbf{2}, \mathbf{2})$.

\section{Acknowledgements}

We are indebted to J. Buchbinder, S. Fedoruk, M. Jardim, A. Nersessian, V. Roubtsov, I. Smilga, S. Theisen, and, especially, to A.Wipf for useful discussions and correspondence. The work of E.I. was supported in part by RFBR grants 09-02-01209, 09-01-93107, 09-02-91349 and a grant of the Heisenberg-Landau program. He thanks SUBATECH, Université de Nantes, for the kind hospitality in the course of this study.

\section{References}

[1] A physicist may consult [T. Eguchi, P.B. Gilkey, and A.J. Hanson, Phys. Repts. 66 (1980) 213].

[2] M.F. Atiyah and I.M. Singer, Annals Math. 87 (1968) 484.

[3] M.F. Atiyah and I.M. Singer, Annals Math. 87 (1968) 546. 
[4] M.F. Atiyah and I.M. Singer, Annals Math. 93 (1971) 119.

[5] M.F. Atiyah and I.M. Singer, Annals Math. 93 (1971) 139.

[6] B. Zumino, Supersymmetry and the index theorem Preprint LBL-17972, UCBPTH-84-17 (Lecture at the Shelter Island Conference, Shelter Island, NY, June 1983).

[7] J. Mañes and B.Zumino, Nucl. Phys. B270 (1986) 651.

[8] A.S. Schwarz, Phys. Lett. 67B (1977) 172.

[9] R. Jackiw and C. Rebbi, Phys. Lett. 67B (1977) 189.

[10] C. Callan, R. Dashen, and D. Gross, Phys. Rev. D17 (1978) 2717.

[11] See e.g. [A.V. Smilga, Lectures on Quantum Chromodynamics, World Scientific 2001, Chapt. 12] for detailed explanations.

[12] E. Witten, Nucl. Phys. B188 (1981) 513.

[13] E. Witten, J. Diff. Geom. 17 (1982) 661.

[14] L. Alvarez-Gaume, Commun. Math. Phys. 90 (1983) 161

[15] D. Friedan and P. Windey, Nucl. Phys. B235 (1984) 395.

[16] P. Windey, Acta Phys. Polon. B15 (1984) 435.

[17] A. Mostafazadeh, J. Math. Phys. 35 (1994) 1095, arXiv:hep-th/9309060.

[18] A. Mostafazadeh, J. Math. Phys. 35 (1994) 1125, arXiv:hep-th/9309061.

[19] A. Hietamaki, A.Yu. Morozov, A.J. Niemi, and K. Palo, Phys. Lett. B263 (1991) 417.

[20] C.M. Hull arXiv:hep-th/9910028.

[21] A. Kirchberg, J.D. Lange, and A. Wipf, Ann. Phys. 315 (2005) 467, arXiv:hep-th/0401134.

[22] E.A. Ivanov and A.V. Smilga, arXiv:1012.2069v1 [hep-th].

[23] A.V. Smilga, SIGMA 7 (2011) 105, arXiv:1105.3935 [math-ph].

[24] A.V. Smilga, J. Math. Phys. 53 (2012) 042103, arXiv:1104.3986 [math-ph].

[25] A.V. Smilga, SIGMA 8 (2012) 003, arXiv:1109.2867 [math-ph].

[26] S.A. Fedoruk, E.A. Ivanov, and A.V. Smilga, arXiv:1204.4105 [hep-th].

[27] J.-M. Bismut, Math. Ann. 284 (1989) 681. 
[28] N.E. Mavromatos, J. Phys. A21 (1988) 2279.

[29] E. D’Hoker and L. Vinet, Phys. Lett. B137 (1984) 72.

[30] V. P. Akulov and A. I. Pashnev, Theor. Math. Phys. 65 (1985) 1027 [Teor. Mat. Fiz. 65 (1985) 84].

[31] G. A. Mezincescu and L. Mezincescu, J. Math. Phys. 44 (2003) 3595, arXiv:hep-th/0109002.

[32] A.V. Smilga, Nucl. Phys. B292 (1987) 363.

[33] H.J. Groenewold, Physica 12 (1946), pp. 405-460.

[34] I.E. Moyal, Proc. Cambr. Phil. Soc. 45 (1949) 99.

[35] P.S. Howe and G. Papadopoulos, Phys. Lett. B379 (1996) 80.

[36] F. Delduc and E. Ivanov, Nucl. Phys. B855 (2012) 815, arXiv:1107.1429[hep-th].

[37] H. Braden, Ann. Phys. NY 171 (1986) 433.

[38] M.A. Konyushikhin and A.V. Smilga, Phys. Lett. B689 (2010) 95, arXiv:0910.5162 [hep-th].

[39] E. Ivanov and O. Lechtenfeld, JHEP 0309 (2003) 073, arXiv:hep-th/0307111.

[40] E.A. Ivanov, M.A. Konyushikhin, and A.V. Smilga, JHEP 1005 (2010) 033, arXiv:0912.3289 [hep-th].

[41] B. Zumino, Phys. Lett. 87B (1984) 203.

[42] A.C. Davis, A.J. Macfarlane, P.Popat, and J.W. van Holten, J. Phys. A17 (1984) 2945.

[43] A.J. Macfarlane and P.C. Popat, J. Phys. A17 (1984) 2955.

[44] P. di Vecchia and S. Ferrara, Nucl. Phys. B130 (1977) 93.

[45] E. Witten, Phys. Rev. D16 (1977) 2991.

[46] D.Z. Freedman and P.K. Townsend, Nucl. Phys. B177 (1981) 282.

[47] E. Ivanov, S. Krivonos, and O. Lechtenfeld, Class. Quant. Grav. 21 (2004) 1031, arXiv:hep-th/0310299.

[48] S. Bellucci, S. Krivonos, A. Marrani, and E. Orazi, Phys. Rev. D73 (2006) 025011, arXiv:hep-th/0511249.

[49] L.I. Nicolaescu, Notes on Seiberg-Witten theory, AMS, Providence, 2000. 
[50] A.V. Smilga, arXiv:1209.0539 [math-ph].

[51] S. Cecotti and L. Girardello, Phys. Lett. B110 (1982) 39.

[52] L. Girardello, C. Imbimbo, and S. Mukhi., Phys. Lett. B132 (1983) 69.

[53] E. Witten, in [Shifman M.A., ed.: The many faces of the superworld, World Scientific, Singapore, 2000, p.156], arXiv:hep-th/9903005.

[54] A.V. Smilga, JHEP 1001 (2010) 086, arXiv:0910.0803 [hep-th].

[55] A.V. Smilga, JHEP 1205 (2012) 103, arXiv:1202.6566 [hep-th].

[56] E. Ivanov, L. Mezincescu, and P.K. Townsend, in [Salamanca 2003, Symmetries in gravity and field theory, p.385], arXiv:hep-th/0311159.

[57] A.V. Smilga, Commun. Math. Phys. 230 (2002) 245, arXiv:hep-th/0110105.

[58] S. Fubini and E. Rabinovici, Nucl. Phys. B245 (1984) 17.

[59] A.V. Smilga, Nucl. Phys. B266 (1986) 45.

[60] P. Yi, Nucl. Phys. B505 (1997) 307, arXiv:hep-th/9704098.

[61] S. Sethi and M. Stern, Commun. Math. Phys. 194 (1998) 675, arXiv:hep-th/9705046.

[62] G. Moore, N. Nekrasov, and S. Shatashvili, Commun. Math. Phys. 209 (2000) 77, arXiv:hep-th/9803265.

[63] V.G. Kac and A.V. Smilga, Nucl. Phys. B571 (2000) 515, arXiv:hep-th/9908096.

[64] A.V. Smilga, JETP 64 (1986) 8.

[65] B. Yu. Blok and A.V. Smilga, Nucl. Phys. B287 (1987) 589.

[66] A.V. Smilga, Nucl. Phys. B291 (1987) 241.

[67] E.A. Ivanov and A.V. Smilga, Phys. Lett. B257 (1991) 79.

[68] V.P. Berezovoj and A.I. Pashnev, Class. Quant. Gravity 8 (1991) 2141. 